\title{
HOMOGENIZATION OF THE NEUMANN PROBLEM IN PERFORATED DOMAINS: AN ALTERNATIVE APPROACH
}

\author{
MARCO BARCHIESI \& MATTEO FOCARDI
}

\begin{abstract}
The main goal of this paper is a compactness result for families of functions in the space $S B V$ (Special functions of Bounded Variation) defined on periodically perforated domains. Given an open and bounded set $\Omega \subseteq \mathbb{R}^{n}$, and an open, connected, and $(-1 / 2,1 / 2)^{n}$-periodic set $P \subseteq \mathbb{R}^{n}$, consider for any $\varepsilon>0$ the perforated domain $\Omega_{\varepsilon}:=\Omega \cap \varepsilon P$. Let $\left(u_{\varepsilon}\right) \subset$ $S B V^{p}\left(\Omega_{\varepsilon}\right), p>1$, be such that $\int_{\Omega_{\varepsilon}}\left|\nabla u_{\varepsilon}\right|^{p} d x+\mathcal{H}^{n-1}\left(S_{u_{\varepsilon}} \cap \Omega_{\varepsilon}\right)+\left\|u_{\varepsilon}\right\|_{L^{p}\left(\Omega_{\varepsilon}\right)}$ is bounded. Then, we prove that, up to a subsequence, there exists $u \in$ $G S B V^{p} \cap L^{p}(\Omega)$ satisfying $\lim _{\varepsilon}\left\|u-u_{\varepsilon}\right\|_{L^{1}\left(\Omega_{\varepsilon}\right)}=0$.

Our analysis avoids the use of any extension procedure in $S B V$, weakens the hypothesis on $P$ to minimal ones and simplifies the proof of the results recently obtained in $[18,14]$. Among the arguments we introduce, we provide a localized version of the Poincaré-Wirtinger inequality in $S B V$. As an application we study the asymptotic behavior of a brittle porous material represented by the perforated domain $\Omega_{\varepsilon}$.

Finally, we slightly extend the well known homogenization theorem for Sobolev energies on perforated domains.
\end{abstract}

Keywords: compactness in $S B V$, free discontinuity, homogenization, perforated domain.

2000 Mathematics Subject Classification: 35B27, 73B27, 73M25

\section{Contents}

1. Introduction $\quad 1$

2. $S B V^{p}$ compactness 4

3. Lusin type approximation 16

4. Homogenization $r$

$\begin{array}{lr}\text { Appendix A. } & 27\end{array}$

$\begin{array}{lr}\text { References } & 29\end{array}$

\section{INTRODUCTION}

A new technique for the homogenization of Neumann problems in periodic perforated domains in the class $S B V$ is the main subject of this paper.

Such a problem arises in a variational model for brittle porous media, as recently studied in [18]. The model is obtained as the homogenization limit of hyperelastic brittle bodies $\Omega_{\varepsilon}$ whose material parts are the intersection of an open set $\Omega \subseteq \mathbb{R}^{2}$ and of the $\varepsilon$-scaled

Date: February 15, 2010. 
copy $\varepsilon P$ of a $Q$-periodic, connected, open set $P \subseteq \mathbb{R}^{2}$. with $Q=(-1 / 2,1 / 2)^{2}$ denoting the unit cube. The perforation is represented by $\mathbb{R}^{2} \backslash \varepsilon P$.

In the case of antiplane shear, $\Omega_{\varepsilon}:=\Omega \cap \varepsilon P$ is a section of the reference configuration of the cylindrical body $\Omega_{\varepsilon} \times \mathbb{R}$. According to the weak formulation of Griffith's theory of brittle fracture introduced by Ambrosio and Braides [4], it is assumed that the (component in the direction perpendicular to $\Omega_{\varepsilon}$ of the) displacement $u$ belongs to the class $S B V\left(\Omega_{\varepsilon}\right)$ of Special functions with Bounded Variation. By adopting this functional framework the crack site is identified by the set $S_{u}$ of (approximate) discontinuities of $u$, which is an $\mathcal{H}^{1}$ rectifiable set. The orientation of the crack is then described by the normal $\nu_{u}$ to $S_{u}$. Under suitable boundary conditions, the equilibrium configurations of the system are reached by minimizing the sum of the elastic energy stored in the uncracked part of the body, and the surface energy dissipated to enlarge the crack. To take into account possible inhomogeneities and anisotropies of the material the total energy then takes the form:

$$
\mathcal{F}_{\varepsilon}(u):=\int_{\Omega_{\varepsilon}} f\left(\frac{x}{\varepsilon}, \nabla u(x)\right) d x+\int_{S_{u} \cap \Omega_{\varepsilon}} g\left(\frac{x}{\varepsilon}, \nu_{u}(x)\right) d \mathcal{H}^{1},
$$

where $f: \mathbb{R}^{2} \times \mathbb{R}^{2} \rightarrow[0,+\infty)$ and $g: \mathbb{R}^{2} \times \mathbb{S}^{1} \rightarrow[0,+\infty)$ are Carathéodory integrands, $Q$-periodic in the first variable and satisfying suitable growth assumptions (see Section 4).

In this variational framework the asymptotic analysis as $\varepsilon \rightarrow 0^{+}$of the energies $\mathcal{F}_{\varepsilon}$ can be performed in terms of $\Gamma$-convergence (for its definition and basic properties we refer to [8]). Indeed, when suitable boundary conditions are imposed, the minimizers of $\mathcal{F}_{\varepsilon}$ converge to those of the related minimum problem for the $\Gamma$-limit.

Due to the presence of the holes, a major difficult task that arises is to characterize the domain of the possible $\Gamma$-limits, or better to study the compactness properties of families $\left(u_{\varepsilon}\right)$ with equibounded energies, i.e., $\sup _{\varepsilon} \mathcal{F}_{\varepsilon}\left(u_{\varepsilon}\right)<\infty$.

From a mathematical point of view this question is interesting not only in the 2dimensional setting mentioned so far. Hence, in the following we turn to the more general $n$-dimensional setting; relevant quantities are then supposed to be redefined accordingly.

Due to the assumptions on the densities $f$ and $g$ above (see Section 4 for the details) the sequence $\left(u_{\varepsilon}\right)$ is bounded in $S B V^{p}\left(\Omega_{\varepsilon}\right), p \in(1,+\infty)$, that is

$$
\sup _{\varepsilon}\left(\int_{\Omega_{\varepsilon}}|\nabla u(x)|^{p} d x+\mathcal{H}^{n-1}\left(S_{u} \cap \Omega_{\varepsilon}\right)\right)<\infty .
$$

With an additional bound on $\left\|u_{\varepsilon}\right\|_{L^{\infty}\left(\Omega_{\varepsilon}\right)}$, in [18] it has been proved that suitable $L^{\infty}$ extensions of $u_{\varepsilon}$ to all of $\Omega$ converge (up to subsequences) in $L^{1}(\Omega)$ to functions in $S B V^{p}(\Omega)$. The method introduced in [18] consists of two main steps: first, provide extensions $\left(\tilde{u}_{\varepsilon}\right)$ having $L^{1}$ limit $u$ in $B V(\Omega)$, and then upgrade the regularity of the limit to $S B V^{p}(\Omega)$. This upgrade is achieved in dimension two via a careful truncation argument (see [18, Lemma 4.2 and Theorem 4.1] and the comments before Proposition 2), and it is obtained in higher dimensions thanks to a slicing technique. The previous approach can be carried out only under the restriction $Q \backslash P \subset Q$, i.e., the reference hole $Q \backslash P$ is far from the boundary of $Q$. Indeed, the idea in [18] is to perform in an annulus around each perforation a careful truncation so that the truncated function $v_{\varepsilon}$ has on such annuli oscillation controlled in terms of the $L^{p}$ norm of $\nabla u_{\varepsilon}$ (see [18, Subsection 4.2]). In view of this Poincaré-Wirtinger type inequality, an $S B V^{p}$ extension $\tilde{v}_{\varepsilon}$ of $v_{\varepsilon}$ to the whole $\Omega$, with controlled energies, is obtained by means of a standard cut-off technique. Finally, the compactness criterion in $S B V^{p}$ implies that $\left(\tilde{v}_{\varepsilon}\right)$ still converges to $u$ and $u \in S B V^{p}(\Omega)$. 
Such result has been subsequently proved in [14] under the only assumption that $P$ is connected and Lipschitz by using a different technique: the construction of a family of equibounded extension operators from $S B V^{p}\left(\Omega_{\varepsilon}\right)$ to $S B V^{p}(\Omega)$ (see [14, Theorem 1.3]). The construction is carried out first by extending $u_{\varepsilon}$ in a small neighborhood of $\Omega \cap \partial(\varepsilon P)$, on which the function is regularized afterwards thanks to a powerful tool to investigate the regularity properties of local minimizers of free-discontinuity problems: the so called density lower bound or elimination property of the jump set (see [5, Theorem 7.21]). Such regularity result shows that in case the proportion of the jump set of a local minimizer in a ball is below a dimensional threshold, there is actually no jump in the concentric ball with half radius. The extension procedure is completed to all $\Omega$ by applying the techniques developed for the Sobolev case in [1, Theorem 2.1] to the regularization of $u_{\varepsilon}$ around $\Omega \cap \partial(\varepsilon P)$.

The main goal of this paper is to give an alternative and more elementary approach to the $S B V^{p}$ compactness issue mentioned above. We do not try to fill the holes of $\Omega_{\varepsilon}$ to obtain an $S B V^{p}$ extension of $u_{\varepsilon}$ to all $\Omega$, but rather we use $u_{\varepsilon}$ as a frame to create new functions $v_{\varepsilon}$ in $S B V^{p}(\Omega)$ having the same asymptotic behavior. For that, we need just to require that $P$ is connected.

Assuming $\left\|u_{\varepsilon}\right\|_{L^{p}\left(\Omega_{\varepsilon}\right)}$ equibounded, a brief outline is as follows: first, we define a suitable $L^{p}$ extension $\tilde{u}_{\varepsilon}$ of $u_{\varepsilon}$ to all $\Omega$ and we show that $\left(\tilde{u}_{\varepsilon}\right)$ is strongly convergent in $L^{1}(\Omega)$ to some function $u$. In this part our approach follows that by Allaire and Murat [2] relying on the M. Riesz-Fréchet-Kolmogorov compactness criterion.

As a second step, with fixed a ball $B_{r} \subseteq Q \backslash P$, we modify $u_{\varepsilon}$ to make it constant in each ball $\left(B_{r}\right)_{\varepsilon}^{i}:=\varepsilon\left(B_{r}+i\right), i \in \mathbb{Z}^{n}$, included in $\Omega$. To this aim, the main ingredient is Proposition 2, a localized version of the Poincaré-Wirtinger type inequality in $S B V$ by De Giorgi, Carriero and Leaci (see [15, Theorem 3.1] and [5, Theorem 4.14]). The latter result is the starting point of the regularity topics for free-discontinuity problems quoted before. We think that Proposition 2 may be of some interest in itself.

The oscillation of the constant values of $u_{\varepsilon}$ on the balls $\left(B_{r}\right)_{\varepsilon}^{i}$ can be essentially estimated in term of the $L^{p}$ norm of $\nabla u_{\varepsilon}$, being $P$ connected and $\mathcal{H}^{n-1}\left(S_{u_{\varepsilon}} \cap \Omega_{\varepsilon}\right)$ bounded. Finally, we employ a linear interpolation argument to construct functions $v_{\varepsilon}$ in $S B V^{p}(\Omega)$ having the same constant values of $u_{\varepsilon}$ on the balls $\left(B_{r}\right)_{\varepsilon}^{i}$, the same $L^{1}(\Omega)$ limit $u$, and bounded energies on $\Omega$. The classical compactness criterion in $S B V^{p}$ then implies that $u$ is in $G S B V^{p}(\Omega)$.

A more detailed description of our strategy is postponed to Section 2 after the statement of the compactness result Theorem 1.

In view of the compactness properties established in Theorem 1 we can determine the $\Gamma$-limit $\mathcal{F}_{\text {hom }}$ of $\left(\mathcal{F}_{\varepsilon}\right)$ with respect to the strong $L^{p}$ topology. In particular, we show that the proof of [18, Theorem 5.1] can be suitably modified to work also in the case under scrutiny. In addition, we change some arguments originally employed in $[18$, Proposition 5.3] to avoid the extension results [1, Theorem 2.1], and to make the proof self-contained. Moreover, we fix a technical difficulty overlooked in [18, Step 2 of Proposition 5.3] and related to a truncation technique contained in [21, Lemma 2.1].

As a byproduct, we derive an alternative proof of the classical homogenization result for energies defined on Sobolev spaces in perforated domains, i.e., $\mathcal{F}_{\varepsilon}$ in (1.1) restricted to $W^{1, p}\left(\Omega_{\varepsilon}\right)$. Our result is stated under assumptions slightly weaker than those in $[1$, Theorems 3.1 and 3.6] (see also [10, Theorem 3.1]), and avoids the use of any extension procedure in $W^{1, p}$ (see Theorems 2 and 5). In the linear case, following the oscillating test functions method by Tartar (see [25]), different approaches to this issue have been 
developed, we quote the one by Allaire and Murat [2] related to the M. Riesz-FréchetKolmogorov compactness criterion, and the spectral approach by Briane [12].

The paper is organized as follows: Sections 2 is devoted to the compactness property of sequences with equibounded energies, we prove that their limit are $G S B V$ functions. In Section 3 we adapt the classical Lipschitz approximation of $S B V$ function to the case of perforated domains. In doing that we deal with fine truncation procedures via the maximal operator. Finally, we apply the conclusions of the previous sections to prove the quoted homogenization result in Section 4. In the Appendix we collect some basic results, but maybe less known, employed in what follows.

To conclude the introduction let us recall the principal notations and the functional framework which will be employed throughout the whole paper.

Standard notations are used for the Lebesgue $n$-dimensional measure $\mathcal{L}^{n}$, for the Hausdorff $(n-1)$-dimensional measure $\mathcal{H}^{n-1}$ in $\mathbb{R}^{n}$, for Lebesgue and Sobolev spaces. The average of a function $u$ in $L^{1}(U)$ is indicated by $u_{U}$. Moreover, given a vector $h \in \mathbb{R}^{n}$, we indicate by $\tau_{h}$ the traslation operator: $\tau_{h}(u)(x):=u(x+h)$.

$Q=(-1 / 2,1 / 2)^{n}$ denotes the unit cube in $\mathbb{R}^{n}$ centered in the origin, and $\mathcal{Q}:=(0,1)^{n}$ its translated copy. $B_{r}(x)$ denotes the open ball of radius $r$ centered in $x$. If $x=0$, we simply write $B_{r}$. The set $\mathbb{E}$ denotes the canonical base of $\mathbb{R}^{n}$.

In the whole paper we fix $\Omega$ and $P$ open not empty subsets of $\mathbb{R}^{n}$ such that $\Omega$ is bounded and

$$
P \text { is } Q \text {-periodic and connected. }
$$

In Section 3 we assume in addition $P$ Lipschitz.

Given any set $U \subseteq \mathbb{R}^{n}$, for every $i \in \mathbb{Z}^{n}$ and $\varepsilon>0$ we set $U_{\varepsilon}^{i}:=\varepsilon(U+i)$. Eventually, we define $\Omega_{\varepsilon}:=\Omega \cap \varepsilon P$.

As already mentioned the functional setting of our analysis is that of special functions with bounded variation on $\Omega$, in short $S B V(\Omega)$. We consider also the larger family of the generalized special functions with bounded variation on $\Omega, G S B V(\Omega)$, which is made of all the measurable functions $u: \Omega \rightarrow \mathbb{R}$ whose truncation $u \wedge j \vee-j$ belongs to $S B V(\Omega)$ for every $j \in \mathbb{N}$. In particular, we are involved with the subspaces

$$
\begin{aligned}
S B V^{p}(\Omega) & :=\left\{u \in S B V(\Omega): \nabla u \in L^{p}(\Omega) \text { and } \mathcal{H}^{n-1}\left(S_{u}\right)<+\infty\right\}, \\
G S B V^{p}(\Omega) & :=\left\{u \in G S B V(\Omega): \nabla u \in L^{p}(\Omega) \text { and } \mathcal{H}^{n-1}\left(S_{u}\right)<+\infty\right\},
\end{aligned}
$$

where $p \in(1,+\infty)$. We refer to the book [5] for the related theory and relevant results.

\section{2. $S B V^{p}$ COMPACTNESS}

This section is devoted to our main result.

Without loss of generality we assume the origin 0 to belong to $P$, this is clearly true up to a translation. Then, we fix some $r>0$ for which $B_{4 r} \subseteq Q \cap P$.

Theorem 1. Let $\varepsilon_{k} \rightarrow 0^{+}$and let $\left(u_{k}\right)$ be a sequence in $S B V^{p}\left(\Omega_{\varepsilon_{k}}\right)$ such that

$$
\sup _{k}\left(\int_{\Omega_{\varepsilon_{k}}}\left(\left|u_{k}\right|^{p}+\left|\nabla u_{k}\right|^{p}\right) d x+\mathcal{H}^{n-1}\left(S_{u_{k}} \cap \Omega_{\varepsilon_{k}}\right)\right)<+\infty .
$$


Consider the following $L^{p}$ extension of $u_{k}$ to all $\Omega$ :

$$
\tilde{u}_{k}(x):= \begin{cases}u_{k}(x) & \text { if } x \in \Omega_{\varepsilon_{k}}, \\ f_{\left(B_{r}\right)_{\varepsilon_{k}}^{i}} u_{k}(x) d x & \text { if } x \in(Q \backslash P)_{\varepsilon_{k}}^{i} \text { and }\left(B_{r}\right)_{\varepsilon_{k}}^{i} \subseteq \Omega_{\varepsilon_{k}}, \\ 0 & \text { elsewhere in } \Omega .\end{cases}
$$

Then there exists $u \in G S B V^{p} \cap L^{p}(\Omega)$ such that (up to a subsequence not relabeled) $\tilde{u}_{k} \rightarrow u$ strongly in $L^{1}(\Omega)$. Moreover, there is a constant $c=c(n, p, P)$ such that

$$
\begin{gathered}
\int_{\Omega}|\nabla u|^{p} d x \leq c \liminf _{k} \int_{\Omega_{\varepsilon_{k}}}\left|\nabla u_{k}\right|^{p} d x, \\
\mathcal{H}^{n-1}\left(S_{u} \cap \Omega\right) \leq c \liminf _{k} \mathcal{H}^{n-1}\left(S_{u_{k}} \cap \Omega_{\varepsilon_{k}}\right) .
\end{gathered}
$$

Finally, if $\sup _{k}\left\|u_{k}\right\|_{L^{\infty}\left(\Omega_{\varepsilon_{k}}\right)}<+\infty$, then $u \in S B V^{p} \cap L^{\infty}(\Omega)$ and $\tilde{u}_{k} \rightarrow u$ strongly in $L^{p}(\Omega)$.

For the proof of Theorem 1 we need several ingredients, subsumed in the following lemmata.

- Lemma 1, a well known trick that allows to modify a sequence keeping the $L^{1}$ limit.

- Lemma 2, a cut-off argument that allows to modify $\left(u_{k}\right)$, keeping condition (2.1), into a new sequence $\left(w_{k}\right) \subset S B V^{p}\left(\Omega_{\varepsilon_{k}}\right)$ assuming constant values on the balls $\left(B_{r}\right)_{\varepsilon_{k}}^{i}$ and not changing the boundary values on $\left(B_{4 r}\right)_{\varepsilon_{k}}^{i}$. The localized version of the Poincaré-Wirtinger type inequality in $S B V$ contained in Proposition 2 below is instrumental to prove Lemma 2.

- Lemma 3 establishes a delicate estimate of the oscillation of the mean values of a function in $S B V(E)$ in two disjoint balls included in a connected domain $E$, when the jump set is too small to isolate these balls from the rest of the domain.

- Lemma 4 is a simple interpolation argument employed to obtain from $\left(w_{k}\right)$ a piecewise affine sequence $\left(v_{k}\right) \subset S B V^{p}(\Omega)$ taking the same constant values on the balls of radius $r$ centered on vertices of $\mathcal{Q}_{\varepsilon_{k}}^{i}$ mentioned before.

Given Lemmata 1-4 for granted the proof of Theorem 1 can be summarized as follows. Consider $\left(\tilde{u}_{k}\right)$ and $\left(\tilde{w}_{k}\right)$, with $\tilde{w}_{k}$ defined as in (2.2) with $w_{k}$ in place of $u_{k}$, then both sequences turn out to converge (up to subsequences) in $L^{1}(\Omega)$ in view of the M.RieszFréchet-Kolmogorov compactness criterion. In addition, because of Lemma 1 the two sequences have the same limit. To upgrade the regularity of such limit, we prove that $\left(v_{k}\right)$ is weakly pre-compact in $G S B V^{p} \cap L^{p}(\Omega)$. This follows from the estimates in Lemma 3 and Lemma 4 which imply that the $v_{k}$ 's have equi-bounded energy on the whole of $\Omega$. A further application of Lemma 1 shows that $\left(\tilde{u}_{k}\right)$ and $\left(v_{k}\right)$ converge to the same limits.

To begin with we state an elementary result in functional analysis.

Lemma 1. Let $\left(U_{k}\right)$ be a sequence of Borel sets in $\Omega$ such that $\chi_{U_{k}} \rightarrow \theta, \theta \in(0,1]$, weak ${ }^{*}$ in $L^{\infty}(\Omega)$. Given two sequences $\left(u_{k}\right)$ and $\left(v_{k}\right)$ such that $u_{k} \rightarrow u, v_{k} \rightarrow v$ strongly in $L^{1}(\Omega)$, and

$$
u_{k}=v_{k} \quad \mathcal{L}^{n} \text { a.e on } U_{k},
$$

then $u=v \quad \mathcal{L}^{n}$ a.e. on $\Omega$. 
Next we recall the Poincaré-Wirtinger type inequality in $S B V$ proved by De Giorgi, Carriero and Leaci (see [15, Theorem 3.1 and Remark 3.3]). To this aim we need some further notation. Let $u \in S B V\left(B_{4 r}\right)$ be such that

$$
\left(2 \gamma \mathcal{H}^{n-1}\left(S_{u}\right)\right)^{n /(n-1)}<\mathcal{L}^{n}\left(B_{4 r}\right) / 2,
$$

with $\gamma$ the isoperimetric constant, and $n \geq 2$; and define

$$
\begin{aligned}
& \tau_{-}:=\inf \left\{t \in[-\infty,+\infty]: \mathcal{L}^{n}\left(\left\{x \in B_{4 r}: u(x)<t\right\}\right) \geq\left(2 \gamma \mathcal{H}^{n-1}\left(S_{u}\right)\right)^{n /(n-1)}\right\}, \\
& \tau_{+}:=\inf \left\{t \in[-\infty,+\infty]: \mathcal{L}^{n}\left(\left\{x \in B_{4 r}: u(x) \geq t\right\}\right) \leq\left(2 \gamma \mathcal{H}^{n-1}\left(S_{u}\right)\right)^{n /(n-1)}\right\} .
\end{aligned}
$$

Take note that the smallness assumption (2.4) on the jump set of $u$ implies that $\tau_{-} \leq$ $m \leq \tau_{+}$for any median $m$ in $B_{4 r}$ of $u$. Finally, the very definitions of $\tau_{ \pm}$give

$$
\mathcal{L}^{n}\left(\left\{x \in B_{4 r}: u(x)<t\right\}\right) \leq\left(2 \gamma \mathcal{H}^{n-1}\left(S_{u}\right)\right)^{n /(n-1)} \quad \text { for all } t \leq \tau_{-},
$$

and

$$
\mathcal{L}^{n}\left(\left\{x \in B_{4 r}: u(x)>t\right\}\right) \leq\left(2 \gamma \mathcal{H}^{n-1}\left(S_{u}\right)\right)^{n /(n-1)} \quad \text { for all } t \geq \tau_{+} .
$$

The statement below shows that under condition (2.4), the truncation $u \wedge \tau_{+} \vee \tau_{-}$of a function $u$ in $S B V^{p}\left(B_{4 r}\right)$ has mean oscillation from any median of $u$ controlled only in terms of the $L^{p}$ norm of the gradient part of the total variation measure.

Proposition 1. Let $u \in S B V^{p}\left(B_{4 r}\right)$ be satisfying assumption (2.4), for any median $m$ of $u$ it holds

$$
\left\|\left(u \wedge \tau_{+} \vee \tau_{-}\right)-m\right\|_{L^{q}\left(B_{4 r}\right)} \leq \frac{2 \gamma q(n-1)}{n}\left(\mathcal{L}^{n}\left(B_{4 r}\right)\right)^{\frac{1}{n}+\frac{1}{q}-\frac{1}{p}}\|\nabla u\|_{L^{p}\left(B_{4 r}\right)},
$$

for any $q \in[1, n p /(n-p)]$ if $p \in[1, n)$, and $q \in[1,+\infty)$ if $p \in[n,+\infty)$.

Below we prove an enhanced version of Proposition 1 in which we confine the previous truncation argument inside a ball $B_{\varrho}, \varrho \in(2 r, 3 r)$, not to change the boundary values of the given function $u$. Clearly, new discontinuities may arise on $\partial B_{\varrho}$ in this procedure; the main issue is that we are able to control their measure through the measure of the jump set of the original function $u$.

In the 2-dimensional case a different truncation technique has been performed in [18, Lemmata 4.1, 4.2, and Theorem 4.1] (see also [6, Lemma 3.3] for related results). In that setting a capacitary argument shows that actually no new jump arises when truncating, provided the starting function has discontinuity set of sufficiently small measure. Such a result is of genuine 2-dimensional nature (see [18, Remark 4.3]). In addition, it seems difficult to employ the same arguments introduced in [18] in higher dimensions. Thus, we infer an analogous conclusion by exploiting directly Proposition 1 (see [18, Subsection 4.2] for a more detailed comparison of the two truncation methods).

Proposition 2. Let $u \in S B V^{p}\left(B_{4 r}\right)$ be satisfying (2.4), then there exists $\varrho \in(2 r, 3 r)$ such that the function

$$
v= \begin{cases}u & \text { on } B_{4 r} \backslash \bar{B}_{\varrho} \\ u \wedge \tau_{+} \vee \tau_{-} & \text {on } B_{\varrho}\end{cases}
$$

belongs to $S B V^{p}\left(B_{4 r}\right)$. In addition,

(i) for any median $m$ of $u$ and for any $q \in[1, n p /(n-p)]$ if $p \in[1, n), q \in[1,+\infty)$ if $p \in[n,+\infty)$ it holds

$$
\|v-m\|_{L^{q}\left(B_{\varrho}\right)} \leq \frac{2 \gamma q(n-1)}{n}\left(\mathcal{L}^{n}\left(B_{4 r}\right)\right)^{\frac{1}{n}+\frac{1}{q}-\frac{1}{p}}\|\nabla u\|_{L^{p}\left(B_{4 r}\right)},
$$


(ii) for some dimensional constant $c_{1}(n)>1$

$$
\mathcal{H}^{n-1}\left(S_{v}\right) \leq c_{1}(n) \mathcal{H}^{n-1}\left(S_{u}\right)
$$

Proof. We suppose the function $u$ to coincide with its precise representative which is specified $\mathcal{H}^{n-1}$ a.e. in $B_{4 r}$ (see [5, Remark 3.79 and Corollary 3.80]). Take also note that, being $\mathcal{L}^{n}\left(S_{u}\right)=0$, for $\mathcal{L}^{1}$ a.e. $\rho \in(0,4 r)$ we have

$$
\mathcal{H}^{n-1}\left(S_{u} \cap \partial B_{\rho}\right)=0 .
$$

In particular, for all such $\rho$ 's $\mathcal{H}^{n-1}$ a.e. point $x \in \partial B_{\rho}$ is a point of approximate continuity for $u$, with value given by $u(x)$ according to our convention.

We use the coarea formula for Lipschitz functions [5, Theorem 2.93] and the mean value theorem to infer the existence of $\varrho \in(2 r, 3 r)$ for which $(2.9)$ holds true and such that

$$
\begin{aligned}
\mathcal{H}^{n-1} & \left(\left\{x \in \partial B_{\varrho}: u(x)<\tau_{-} \text {or } u(x)>\tau_{+}\right\}\right) \\
& \leq \frac{1}{r} \int_{2 r}^{3 r} \mathcal{H}^{n-1}\left(\left\{x \in \partial B_{\rho}: u(x)<\tau_{-} \text {or } u(x)>\tau_{+}\right\}\right) d \rho \\
& \leq \frac{1}{r} \mathcal{L}^{n}\left(\left\{x \in B_{4 r}: u(x)<\tau_{-} \text {or } u(x)>\tau_{+}\right\}\right) \stackrel{(2.5),(2.6)}{\leq} \frac{2}{r}\left(2 \gamma \mathcal{H}^{n-1}\left(S_{u}\right)\right)^{n /(n-1)} \\
& \stackrel{(2.4)}{\leq} \frac{2}{r}\left(\frac{\mathcal{L}^{n}\left(B_{4 r}\right)}{2}\right)^{1 / n} 2 \gamma \mathcal{H}^{n-1}\left(S_{u}\right)=16 \gamma\left(\frac{\mathcal{L}^{n}\left(B_{1}\right)}{2}\right)^{1 / n} \mathcal{H}^{n-1}\left(S_{u}\right) .
\end{aligned}
$$

To prove that the function $v$ in $(2.7)$ is in $S B V\left(B_{4 r}\right)$ it suffices to notice that

$$
v=u \chi_{B_{4 r} \backslash \bar{B}_{\varrho}}+\left(u \wedge \tau_{+} \vee \tau_{-}\right) \chi_{B_{\varrho}}
$$

and each summand regarded as extended to 0 on $\bar{B}_{\varrho}$ and on $B_{4 r} \backslash B_{\varrho}$, respectively, is $S B V\left(B_{4 r}\right)$ regular (see for instance [5, Theorem 3.87, Corollary 3.89]). In addition, we have

$D v=D u\left\llcorner\left(B_{4 r} \backslash \bar{B}_{\varrho}\right)+\left(u_{\text {ext }}-\left(u \wedge \tau_{+} \vee \tau_{-}\right){ }_{\text {int }}\right) \nu_{\partial B_{\varrho}} \mathcal{H}^{n-1}\left\llcorner\partial B_{\varrho}+D\left(u \wedge \tau_{+} \vee \tau_{-}\right)\left\llcorner B_{\varrho}\right.\right.\right.$, with the boundary of the ball $B_{\varrho}$ oriented by the exterior normal $\nu_{\partial B_{\varrho}}$, and with the exterior trace with respect to $\nu_{\partial B_{\varrho}}$ labeled by the subscript ext and the interior one by the subscript int.

By (2.9) it follows that $u_{\text {ext }}(x)=u_{\text {int }}(x)=u(x)$ for $\mathcal{H}^{n-1}$ a.e. $x \in \partial B_{\varrho}$ (see [5, Remark 3.79]), and since $\left(u \wedge \tau_{+} \vee \tau_{-}\right)_{\text {int }}(x)=u_{\text {int }}(x) \wedge \tau_{+} \vee \tau_{-}$for $\mathcal{H}^{n-1}$ a.e. $x \in \partial B_{\varrho}$ we have

$$
S_{v} \subseteq S_{u} \cup\left\{x \in \partial B_{\varrho}: u(x)<\tau_{-} \text {or } u(x)>\tau_{+}\right\}
$$

up to a set of null $\mathcal{H}^{n-1}$ measure. Thus, $v \in S B V^{p}\left(B_{4 r}\right)$, and item (ii) is a consequence of (2.10) by taking the constant $c_{1}(n):=1+16 \gamma\left(\mathcal{L}^{n}\left(B_{1}\right) / 2\right)^{1 / n}$.

To conclude, notice that item (i) follows straightforward from Proposition 1 and the very definition of $v$.

Remark 1. Take note that if $\mathcal{H}^{n-1}\left(S_{u}\right)=0$ then $\tau_{-}=-\infty$ and $\tau_{+}=+\infty$; in particular Proposition 1 and Proposition 2 boil down to the classical Poincaré-Wirtinger inequality in the Sobolev space $W^{1, p}$.

Thanks to Proposition 2 we are able to modify a function $u$ in $S B V^{p}\left(B_{4 r}\right)$ satisfying (2.4) into a new function $w$ in the same class, constant on $B_{r}$, and with gradient and surface energies controlled (separately) in terms of the same quantities of $u$.

Lemma 2. Let $u \in S B V^{p} \cap L^{p}\left(B_{4 r}\right)$ be satisfying (2.4). Then there exists $w \in S B V^{p} \cap$ $L^{p}\left(B_{4 r}\right)$ such that 
(i) $w$ is constant on $B_{r}$;

(ii) $w=u$ on $B_{4 r} \backslash B_{\varrho}$, for some $\varrho \in(2 r, 3 r)$;

(iii) $\|w\|_{L^{p}\left(B_{4 r}\right)} \leq c_{2}\|u\|_{L^{p}\left(B_{4 r}\right)}$;

(iv) $\|\nabla w\|_{L^{p}\left(B_{4 r}, \mathbb{R}^{n}\right)} \leq c_{2}\|\nabla u\|_{L^{p}\left(B_{4 r}, \mathbb{R}^{n}\right)}$;

(v) $\mathcal{H}^{n-1}\left(S_{w}\right) \leq c_{1} \mathcal{H}^{n-1}\left(S_{u}\right)$;

where $c_{1}$ is the dimensional constant in Proposition 2 and $c_{2}=c_{2}(n, p)$.

Proof. Consider the function $v$ provided by Proposition 2, given a radial cut-off $\phi \in$ $C_{0}^{1}\left(B_{4 r},[0,1]\right)$ such that $\left.\phi\right|_{B_{r}}=0,\left.\phi\right|_{B_{4 r} \backslash B_{2 r}}=1$, and $\|\nabla \phi\|_{L^{\infty}\left(B_{4 r}, \mathbb{R}^{n}\right)} \leq 2 / r$ set

$$
w(x):=\phi(x)[v(x)-m]+m,
$$

with $m$ any median of $u$. The function $w$ clearly satisfies (i), (ii) with $\varrho \in(2 r, 3 r)$ the radius in Proposition 2, and (v).

Furthermore, to infer (iv) we note that $\nabla w=\phi \nabla v+(v-m) \nabla \phi$, thus by (2.8) with $q=p$ we get

$$
\begin{aligned}
& \|\nabla w\|_{L^{p}\left(B_{4 r}, \mathbb{R}^{n}\right)} \leq\|\nabla v\|_{L^{p}\left(B_{4 r}, \mathbb{R}^{n}\right)}+\|(v-m) \nabla \phi\|_{L^{p}\left(B_{4 r}, \mathbb{R}^{n}\right)} \\
& \quad \leq\|\nabla u\|_{L^{p}\left(B_{4 r}, \mathbb{R}^{n}\right)}+\|\nabla \phi\|_{L^{\infty}\left(B_{4 r}, \mathbb{R}^{n}\right)}\|v-m\|_{L^{p}\left(B_{2 r} \backslash B_{r}\right)} \\
& \quad \leq\|\nabla u\|_{L^{p}\left(B_{4 r}, \mathbb{R}^{n}\right)}+\frac{4 \gamma p(n-1)}{n} \frac{\left(\mathcal{L}^{n}\left(B_{4 r}\right)\right)^{1 / n}}{r}\|\nabla u\|_{L^{p}\left(B_{4 r}, \mathbb{R}^{n}\right)} .
\end{aligned}
$$

Finally, to infer (iii) it suffices to take into account that any median $m$ in $B_{4 r}$ of $u$ satisfies by its very definition $|m| \leq 2|u|_{B_{4 r}}$, and thus the conclusion follows,

$$
\|w\|_{L^{p}\left(B_{4 r}\right)}^{p} \leq|m|^{p} \int_{B_{2 r}}|1-\varphi(x)| d x+\|v\|_{L^{p}\left(B_{4 r}\right)}^{p} \leq\left(2^{p-n}+1\right)\|u\|_{L^{p}\left(B_{4 r}\right)}^{p} .
$$

The next result gives an estimate on the oscillation of the mean values on disjoint balls of functions in $S B V$ provided the jump set is small enough.

This argument is the counterpart in the $S B V$ framework of [2, Lemma 2.2], where the classical Poincaré-Wirtinger inequality is used to control the oscillation of the mean values of Sobolev functions on adjacent cubes.

Lemma 3. Let $E \subseteq \mathbb{R}^{n}$ be a connected open set and let $B_{r}(x)$ and $B_{r}(y)$ be two well separated balls included in $E$. Then there exist two positive constants $c_{3}$ an $c_{4}$, intrinsically depending only on $E, r, x$ and $y$, with the following property: for each $\varepsilon>0$ and for each $u \in S B V(\varepsilon E)$ such that $\mathcal{H}^{n-1}\left(S_{u}\right) \leq c_{3} \varepsilon^{n-1}$, there holds

$$
\left|u_{\varepsilon B_{r}(x)}-u_{\varepsilon B_{r}(y)}\right| \leq c_{4} \varepsilon^{1-n}\left[|D u|\left(\varepsilon\left(B_{r}(x) \cup B_{r}(y)\right)\right)+\|\nabla u\|_{L^{1}\left(\varepsilon E, \mathbb{R}^{n}\right)}\right] .
$$

Proof. Let $\delta \in(0,1 /(\sqrt{n}))$ and let e be the $n$-th unit vector in $\mathbb{E}$. Define the open rectangle $R_{\delta}:=\left(Q_{\delta} \cup\left(Q_{\delta}+3 \mathrm{e}\right)\right)^{\mathrm{co}}$ and the open set $A_{\delta}:=B_{1} \cup\left(B_{1}+3 \mathrm{e}\right) \cup R_{\delta}$. Since $E$ is connected and $B_{r}(x), B_{r}(y)$ are well separated, we can find a $\delta$, an open set $A \subseteq E$ and a biLipschitz application $\phi: A_{\delta} \rightarrow A$ such that, up to a roto-traslation, $\left.\phi\right|_{B_{1}}$ (resp., $\left.\phi\right|_{B_{1}+3 \mathrm{e}}$ ) is a homothety on $B_{r}(x)$ (resp., $B_{r}(y)$ ). A pictorial idea is given in Figure 1.

Consider the pullback function $w(x):=u(\varepsilon \phi(x))$, then $w$ belongs to $S B V\left(A_{\delta}\right)$ with

$$
\nabla w(x)=\varepsilon \nabla u(\varepsilon \phi(x)) \nabla \phi(x) \quad \mathcal{L}^{n} \text { a.e on } A_{\delta}, \quad S_{w}=(\varepsilon \phi)^{-1}\left(S_{u}\right)
$$




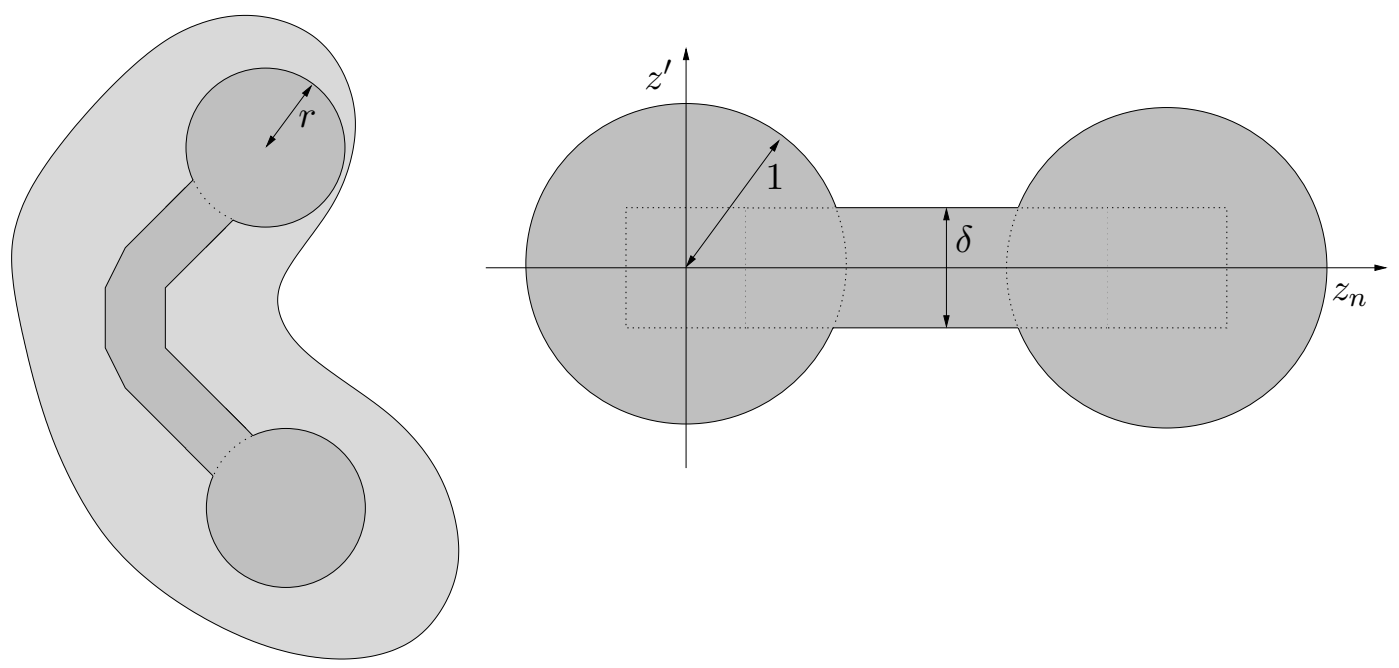

Figure 1 . The sets $A$ and $A_{\delta}$, both in dark gray.

(see [5, Theorem 3.16 and Exercise 4.5]). Hence, we choose $c_{3}$ in such a way that $\mathcal{H}^{n-1}\left(S_{w}\right) \leq \delta^{n-1} / 2$ if $\mathcal{H}^{n-1}\left(S_{u}\right) \leq c_{3} \varepsilon^{n-1}$.

To show (2.11) we argue by a slicing procedure. To this aim we suppose $w$ coinciding with its precise representative defined as in [5, Remark 3.79 and Corollary 3.80]; and we introduce the notation

$$
\begin{aligned}
& Z^{\prime}:=\left\{z^{\prime} \in(-\delta / 2, \delta / 2)^{n-1}:\left(z^{\prime} \times(-\delta / 2,3+\delta / 2)\right) \cap S_{w}=\varnothing\right\}, \\
& Z=Z^{\prime} \times(-\delta / 2, \delta / 2), \quad T_{z}:=\left\{\left(z^{\prime}, z_{n}+\lambda\right): \lambda \in(0,3)\right\},
\end{aligned}
$$

writing $z \in \mathbb{R}^{n}$ as $\left(z^{\prime}, z_{n}\right) \in \mathbb{R}^{n-1} \times \mathbb{R}$. Take note that we have $\mathcal{L}^{n-1}\left(Z^{\prime}\right) \geq \delta^{n-1} / 2$ since $\mathcal{H}^{n-1}\left(S_{w}\right) \leq \delta^{n-1} / 2$. Moreover, by [5, Theorems 3.28, 3.107 and 3.108], for $\mathcal{L}^{n-1}$ a.e. $z^{\prime} \in Z^{\prime}$ the function $w\left(z^{\prime}, \cdot\right)$ is absolutely continuous with derivative given by $\partial_{z_{n}} w\left(z^{\prime}, \cdot\right)$. Then, for $z \in Z$

$$
|w(z)-w(z+3 \mathrm{e})| \leq \int_{T_{z}}\left|\partial_{z_{n}} w\left(z^{\prime}, t\right)\right| d t
$$

and so, by integrating we infer

$$
\left\|w-\tau_{3 \mathrm{e}}(w)\right\|_{L^{1}(Z)} \leq\|\nabla w\|_{L^{1}\left(R_{\delta}, \mathbb{R}^{n}\right)} .
$$

By taking into account that $\mathcal{L}^{n-1}\left(Z^{\prime}\right) \geq \delta^{n-1} / 2$, by using the Poincaré-Wirtinger inequality in $B V(Q)$ in Proposition 5, and inequality (2.12) we get

$$
\begin{aligned}
& \frac{\delta^{n-1}}{2}\left|w_{B_{1}}-w_{B_{1}+3 \mathrm{e}}\right| \leq\left\|w_{B_{1}}-\tau_{3 \mathrm{e}}(w)_{B_{1}}\right\|_{L^{1}(Z)} \\
& \quad \leq\left\|w-w_{B_{1}}\right\|_{L^{1}(Z)}+\left\|w-\tau_{3 \mathrm{e}}(w)\right\|_{L^{1}(Z)}+\left\|\tau_{3 \mathrm{e}}(w)-\tau_{3 \mathrm{e}}(w)_{B_{1}}\right\|_{L^{1}(Z)} \\
& \quad \leq c_{4}\left[|D w|\left(B_{1}\right)+\|\nabla w\|_{L^{1}\left(R_{\delta}, \mathbb{R}^{n}\right)}+|D w|\left(B_{1}+3 \mathrm{e}\right)\right] .
\end{aligned}
$$

Eventually, going back from $w$ to $u$ through $(\varepsilon \phi)^{-1}$ we obtain (2.11).

In the sequel an elementary linear interpolation argument is repeatedly employed to construct a Sobolev function on a neighborhood of a cube with energy controlled in terms of the oscillation of the values on the vertices of the cube and on the size of the cube itself. 
Thus, in Lemma 4 below any extension of a given function is always meant to be obtained via a linear interpolation argument.

Lemma 4. Let $r \in(0,1 / 2)$ and let $p_{1}, \ldots, p_{2^{n}}$ be the vertices of $\varepsilon \mathcal{Q}$. We define $U:=$ $\left(\bigcup_{i=1}^{2^{n}} \mathcal{Q}_{\varepsilon r}\left(p_{i}\right)\right)^{\mathrm{co}}$, where the superscript ${ }^{\text {co }}$ denotes the convex hull and $\mathcal{Q}_{\varepsilon r}\left(p_{i}\right)$ is the cube of size $\varepsilon r$ centered in $p_{i}$. Moreover, given $a_{1}, \ldots, a_{2^{n}} \in \mathbb{R}$, we define $O:=\max \left\{\left|a_{i}-a_{j}\right|\right.$ : $\left.i, j=1, \ldots 2^{n}\right\}$. Then there exists a function $v \in W^{1, p}(U)$ such that $v=a_{i}$ in $\mathcal{Q}_{\varepsilon r}\left(p_{i}\right)$,

$$
\int_{U}|\nabla v|^{p} d x \leq c(n, p, r) O^{p} \varepsilon^{n-p}
$$

and

$$
\int_{U}|v|^{p} d x \leq c(n, p, r, \mathcal{Q})\left(\min _{i}\left|a_{i}\right|^{p}+O^{p}\right) \varepsilon^{n}
$$

Moreover, the values of $v$ along any $(n-1)$-dimensional face of $\varepsilon \mathcal{Q}$ depend only on the values assumed in the vertices belonging to that face.

Proof. Obviously, we start by defining $v=a_{i}$ in $\mathcal{Q}_{\varepsilon r}\left(p_{i}\right)$. Let $L$ be an edge of vertices $p_{i}$ and $p_{j}$. We can extend $v$ to $S(L):=\left(\mathcal{Q}_{\varepsilon r}\left(p_{i}\right) \cup \mathcal{Q}_{\varepsilon r}\left(p_{j}\right)\right)^{\text {co }}$ interpolating the values on the two cubes $\mathcal{Q}_{\varepsilon r}\left(p_{i}\right)$ and $\mathcal{Q}_{\varepsilon r}\left(p_{j}\right)$. Hence, we have the straightforward estimate $|\nabla v|=\left|a_{j}-a_{i}\right| /(\varepsilon-\varepsilon r) \leq 2 O / \varepsilon$, and then

$$
\int_{S(L)}|\nabla v|^{p} d x \leq 2^{p+1} O^{p} r^{n-1} \varepsilon^{n-p}
$$

since $\mathcal{L}^{n}(S(L)) \leq 2 r^{n-1} \varepsilon^{n}$.

Let now $F$ be a $(n-1)$-dimensional face of edges $L_{1}, \ldots, L_{m}$, where $m=2^{n-2}(n-1)$. We can extend $v$ from $\bigcup_{i=1}^{m} S\left(L_{i}\right)$ to $\left(\bigcup_{i=1}^{m} S\left(L_{i}\right)\right)^{\text {co }}$ so to have the $L^{p}$ control of the gradient as in (2.13). This intermediate step ensures that the values of $v$ along a face $F$ depend only on the values assumed in the vertices of the face. It is skipped out in the case $n=2$. Finally, we complete the Sobolev extension to all of $U$.

To conclude we prove (2.14). Suppose $\left|a_{1}\right|=\min _{i}\left|a_{i}\right|$, and apply the Poincaré-Wirtinger inequality in $W^{1, p}$ (see [23, Lemma 1.1.11]) to get

$$
\begin{aligned}
\|v\|_{L^{p}(U)}^{p} & \leq 2^{p-1}\left(\left|a_{1}\right|^{p} \mathcal{L}^{n}(U)+\left\|v-v_{\mathcal{Q}_{\varepsilon r}\left(p_{1}\right)}\right\|_{L^{p}(U)}^{p}\right) \\
& \leq c(n, p, r, \mathcal{Q})\left(\left|a_{1}\right|^{p} \mathcal{L}^{n}(U)+\varepsilon^{p}\|\nabla v\|_{L^{p}\left(U, \mathbb{R}^{n}\right)}^{p}\right)
\end{aligned}
$$

which yields the conclusion if combined with (2.13) and by taking into account that $\mathcal{L}^{n}(U)=(1+2 r)^{n} \varepsilon^{n}$.

We are now ready to prove the $S B V^{p}$ compactness result.

Proof of Theorem 1. Let us first note that the sequence $\left(\tilde{u}_{k}\right)$ is bounded in $L^{p}(\Omega)$ by its very definition and (2.1). Hence, it converges weakly to some function $u$ in $L^{p}(\Omega)$ up to a subsequence not relabeled for convenience. In the sequel we will show that actually $\left(\tilde{u}_{k}\right)$ converges strongly in $L^{1}(\Omega)$, and that $u$ belongs to $G S B V^{p} \cap L^{p}(\Omega)$.

To this aim we initially suppose that $P$ has the cone property with respect to an open cone $C$. Under this assumption we claim that there exists an open, bounded and connected set $E$ having the cone property and such that

$$
P \cap 3 Q \subseteq E \subseteq P .
$$


For, let $F$ be the union of $P \cap 3 Q$ with each congruent copy of $C$ included in $P$ and with vertex in $P \cap 3 Q$. Such a set has the cone property with respect to some cone included in $C$. Then, being bounded, $F$ can be decomposed into a finite number of open sets $F_{1}, \ldots, F_{m}$ each star-shaped with respect to some open ball (see [23, Sezione 1.1.9, Lemma 1]). In particular, the sets $F_{1}, \ldots, F_{m}$ are connected; joining those sets with (finite) chains of congruent copies of $C$ included in $P$, gives the set $E$.

In the following we will often implicitely use that the sets $E_{\varepsilon_{k}}^{i}$ overlap only a finite number of times independently from $i$ and $\varepsilon_{k}$. Throughout steps 1 and 2 below $c$ will denote a positive constant depending (at most) on $n, p, r$ and $E$ which may vary from line to line, unless otherwise stated.

Step 1 . We show that $\left(\tilde{u}_{k}\right)$ is strongly convergent in $L^{1}(\Omega)$.

To this aim we introduce the sets of indexes

$$
I_{k}:=\left\{i \in \mathbb{Z}^{n}: E_{\varepsilon_{k}}^{i} \subseteq \Omega\right\}, \quad J_{k}:=\left\{i \in \mathbb{Z}^{n}: \Omega \cap E_{\varepsilon_{k}}^{i} \neq \varnothing\right\},
$$

and the auxiliary piecewise constant functions

$$
\zeta_{k}(x):= \begin{cases}f_{\left(B_{r}\right)_{\varepsilon_{k}}^{i}} u_{k}(x) d x & \text { if } x \in Q_{\varepsilon_{k}}^{i}, i \in I_{k}, \\ 0 & \text { elsewhere in } \Omega .\end{cases}
$$

We claim that the sequence $\left(\zeta_{k}\right)$ is pre-compact in $L^{1}(\Omega)$. According to the M.RieszFréchet-Kolmogorov compactness criterion (see Theorem IV.25 [11]), being $\left(\zeta_{k}\right)$ bounded in $L^{p}(\Omega)$, it suffices to check that

$$
\lim _{|h| \rightarrow 0} \sup _{k}\left\|\tau_{h}\left(\zeta_{k}\right)-\zeta_{k}\right\|_{L^{1}(A)}=0
$$

for every open subset $A \subset \Omega$. Given this choice, let $h \in \mathbb{R}^{n}$ be such that $|h|<d\left(A, \Omega^{c}\right)$. Notice that for $k \geq k(h)$ the translates $A+h^{\prime}$ are included in $\cup_{i \in I_{k}} E_{\varepsilon_{k}}^{i}$ for every $h^{\prime} \in \mathbb{R}^{n}$ with $\left|h^{\prime}\right| \leq|h|$.

Let us first assume that $h=s \mathrm{e}$, e $\in \mathbb{E}$, and without loss of generality suppose $s \in$ $[0,+\infty)$. We distinguish two cases: either $s \in\left[0, \varepsilon_{k}\right]$ or $s>\varepsilon_{k}$. In the former we split $(Q \cap P)_{\varepsilon_{k}}^{i}, i \in I_{k}$, into the subsets

$U_{i, \varepsilon_{k}}=\left\{x \in(Q \cap P)_{\varepsilon_{k}}^{i}: x+s \mathrm{e} \in(Q \cap P)_{\varepsilon_{k}}^{i}\right\}, \quad V_{i, \varepsilon_{k}}=\left\{x \in(Q \cap P)_{\varepsilon_{k}}^{i}: x+s \mathrm{e} \notin(Q \cap P)_{\varepsilon_{k}}^{i}\right\}$.

By the very definition of $\zeta_{k}$ it follows for $x \in U_{i, \varepsilon_{k}}$

$$
\left|\tau_{s \mathrm{e}}\left(\zeta_{k}\right)(x)-\zeta_{k}(x)\right|=0 .
$$

Furthermore, for any $i \in I_{k}$ both the balls $\left(B_{r}\right)_{\varepsilon_{k}}^{i}$ and $\left(B_{r}\right)_{\varepsilon_{k}}^{i+\mathrm{e}}$ are contained in $E_{\varepsilon_{k}}^{i}$. Then, we can apply Proposition 5 on $E_{\varepsilon_{k}}^{i}$ with respect to $\left(B_{r}\right)_{\varepsilon_{k}}^{i}$ and $\left(B_{r}\right)_{\varepsilon_{k}}^{i+e}$ to obtain for $x \in V_{i, \varepsilon_{k}}$

$$
\begin{aligned}
& \varepsilon_{k}^{n} \mathcal{L}^{n}(E)\left|\zeta_{k}(x)-\tau_{s \mathrm{e}}\left(\zeta_{k}\right)(x)\right|=\left\|\zeta_{k}(x)-\zeta_{k}\left(x+\varepsilon_{k} \mathrm{e}\right)\right\|_{L^{1}\left(E_{\varepsilon_{k}}^{i}\right)} \\
& \quad \leq\left\|u_{k}-\zeta_{k}(x)\right\|_{L^{1}\left(E_{\varepsilon_{k}}^{i}\right)}+\left\|u_{k}-\zeta_{k}\left(x+\varepsilon_{k} \mathrm{e}\right)\right\|_{L^{1}\left(E_{\varepsilon_{k}}^{i}\right)} \leq c \varepsilon_{k}\left|D u_{k}\right|\left(E_{\varepsilon_{k}}^{i}\right) .
\end{aligned}
$$

Notice that $\mathcal{L}^{n}\left(V_{i, \varepsilon_{k}}\right) \leq \varepsilon_{k}^{n-1} s$, by integrating $\left|\zeta_{k}-\tau_{s \mathrm{e}}\left(\zeta_{k}\right)\right|$ over $(Q \cap P)_{\varepsilon_{k}}^{i}$ and by summing up on $i \in I_{k}$, we infer from (2.16) and (2.17), that

$$
\left\|\tau_{s \mathrm{e}}\left(\zeta_{k}\right)-\zeta_{k}\right\|_{L^{1}(A)} \leq c s\left|D u_{k}\right|\left(\Omega_{\varepsilon_{k}}\right) .
$$


Let us now assume that $s>\varepsilon_{k}$, and rewrite $s=m \varepsilon_{k}+s^{\prime}$ with $s^{\prime} \in\left[0, \varepsilon_{k}\right)$ and $m \in \mathbb{N}$. Then, since $\tau_{h^{\prime}} \circ \tau_{h^{\prime \prime}}=\tau_{h^{\prime}+h^{\prime \prime}}$, the argument leading to (2.18) yields

$$
\begin{aligned}
& \left\|\tau_{s \mathrm{e}}\left(\zeta_{k}\right)-\zeta_{k}\right\|_{L^{1}(A)} \leq \sum_{r=0}^{m-1}\left\|\tau_{(r+1) \varepsilon_{k} \mathrm{e}}\left(\zeta_{k}\right)-\tau_{r \varepsilon_{k} \mathrm{e}}\left(\zeta_{k}\right)\right\|_{L^{1}(A)} \\
& \quad+\left\|\tau_{s \mathrm{e}}\left(\zeta_{k}\right)-\tau_{m \varepsilon_{k} \mathrm{e}}\left(\zeta_{k}\right)\right\|_{L^{1}(A)} \leq c\left(m \varepsilon_{k}+s^{\prime}\right)\left|D u_{k}\right|\left(\Omega_{\varepsilon_{k}}\right),
\end{aligned}
$$

from which in turn we deduce that (2.18) holds true also in case $s>\varepsilon_{k}$.

Let now $h$ be any vector in $\mathbb{R}^{n}$ with $|h|<\operatorname{dist}\left(A, \Omega^{c}\right)$, by varying one coordinate per time and fixing all the others, we deduce from (2.18)

$$
\left\|\tau_{h}\left(\zeta_{k}\right)-\zeta_{k}\right\|_{L^{1}(A)} \leq c|h|\left|D u_{k}\right|\left(\Omega_{\varepsilon_{k}}\right)
$$

with $c$ a positive constant depending neither on $h$ nor on $A$. From (2.1) and (2.19) we get (2.15), i.e., $\left(\zeta_{k}\right)$ is pre-compact in $L^{1}(\Omega)$.

Let us prove that $\left(\tilde{u}_{k}\right)$ is pre-compact in $L^{1}(\Omega)$, too. By Proposition 5 we have

$$
\begin{aligned}
\left\|\tilde{u}_{k}-\zeta_{k}\right\|_{L^{1}(\Omega)} & \leq \sum_{i \in I_{k}} \int_{E_{\varepsilon_{k}}^{i}}\left|u_{k}-f_{\left(B_{r^{\prime}}\right)_{\varepsilon_{k}}^{i}} u_{k}(y) d y\right| d x+c \int_{\cup_{i \in J_{k} \backslash I_{k}}\left(E_{\varepsilon_{k}}^{i} \cap \Omega\right)}\left|u_{k}\right| d x \\
& \leq c \varepsilon_{k} \sum_{i \in I_{k}}\left|D u_{k}\right|\left(E_{\varepsilon_{k}}^{i}\right)+c \int_{\cup_{i \in J_{k} \backslash I_{k}}\left(E_{\varepsilon_{k}}^{i} \cap \Omega\right)}\left|u_{k}\right| d x \\
& \leq c \varepsilon_{k}\left|D u_{k}\right|\left(\Omega_{\varepsilon_{k}}\right)+c \mathcal{L}^{n}\left(\cup_{i \in J_{k} \backslash I_{k}}\left(E_{\varepsilon_{k}}^{i} \cap \Omega\right)\right)^{\frac{p-1}{p}}\left\|u_{k}\right\|_{L^{p}\left(\Omega_{\varepsilon_{k}}\right)} .
\end{aligned}
$$

Then, since $\lim _{k} \mathcal{L}^{n}\left(\cup_{i \in J_{k} \backslash I_{k}}\left(E_{\varepsilon_{k}}^{i} \cap \Omega\right)\right)=0$, by (2.1) it follows that $\lim _{k}\left\|\tilde{u}_{k}-\zeta_{k}\right\|_{L^{1}(\Omega)}=0$.

Step 2. We prove that the strong $L^{1}$ limit $u$ of $\left(\tilde{u}_{k}\right)$ belongs to $G S B V^{p} \cap L^{p}(\Omega)$.

Let $H_{k}:=\left\{i \in I_{k}: \mathcal{H}^{n-1}\left(S_{u_{k}} \cap E_{\varepsilon_{k}}^{i}\right) \leq \delta \varepsilon_{k}^{n-1}\right\}$, with $\delta=\delta(n, r, E)>0$ small enough in order to apply both Lemmata 2 and 3 . Take note that the number of elements in $I_{k} \backslash H_{k}$ is bounded by $\delta^{-1} \varepsilon^{1-n} \mathcal{H}^{n-1}\left(S_{u_{k}} \cap \Omega_{\varepsilon_{k}}\right)$, then by $(2.1)$ we infer $\lim _{k} \mathcal{L}^{n}\left(\cup_{i \in J_{k} \backslash H_{k}}\left(E_{\varepsilon_{k}}^{i} \cap \Omega\right)\right)=$ 0 . In each $E_{\varepsilon_{k}}^{i}, i \in H_{k}$, we modify $u_{k}$ on all the balls of radius $4 r \varepsilon_{k}$ centered in the vertices of the cube $\mathcal{Q}_{\varepsilon_{k}}^{i}$ by exploiting Lemma 2, and define the function $w_{k}$ accordingly. Then, by construction $w_{k}$ assumes constant values on the concentric balls of radius $r \varepsilon_{k}$ (see Figure 2).

In view of items (iii)-(v) in Lemma 2, we get for each $i \in H_{k}$

$$
\begin{gathered}
\int_{E_{\varepsilon_{k}}^{i}}\left|w_{k}\right|^{p} d x \leq c \int_{E_{\varepsilon_{k}}^{i}}\left|u_{k}\right|^{p} d x, \quad \int_{E_{\varepsilon_{k}}^{i}}\left|\nabla w_{k}\right|^{p} d x \leq c \int_{E_{\varepsilon_{k}}^{i}}\left|\nabla u_{k}\right|^{p} d x, \\
\mathcal{H}^{n-1}\left(S_{w_{k}} \cap E_{\varepsilon_{k}}^{i}\right) \leq c \mathcal{H}^{n-1}\left(S_{u_{k}} \cap E_{\varepsilon_{k}}^{i}\right),
\end{gathered}
$$

so that $\left(w_{k}\right)$ still satisfies the energy bound (2.1).

Let $\tilde{w}_{k}$ be the $L^{p}$ extension of $w_{k}$ to all of $\Omega$ defined in (2.2), with $w_{k}$ in place of $u_{k}$. Repeating the argument employed for $\left(\tilde{u}_{k}\right)$, we have that $\left(\tilde{w}_{k}\right)$ is pre-compact in $L^{1}(\Omega)$. Moreover, since $\tilde{u}_{k}$ and $\tilde{w}_{k}$ coincide (at least) on the set $\cup_{i \in H_{k}}\left(B_{4 r}\right)_{\varepsilon_{k}}^{i} \backslash\left(B_{3 r}\right)_{\varepsilon_{k}}^{i}$, Lemma 1 entails that they have the same cluster points.

Next we modify $\left(w_{k}\right)$ in order to get a sequence $\left(v_{k}\right)$ pre-compact in $G S B V^{p}(\Omega)$. First, we apply Lemma 4 in each cube $\mathcal{Q}_{\varepsilon_{k}}^{i}, i \in H_{k}$, by using the constant values of $w_{k}$ on the balls of radius $r \varepsilon_{k}$ and centered in the vertices of such cubes and define $v_{k}$ accordingly. Eventually, we set $v_{k}$ equal to 0 on $\Omega \backslash \cup_{i \in H_{k}} \mathcal{Q}_{\varepsilon_{k}}^{i}$. A pictorial idea of the construction of the function $v_{k}$ is given in Figure 3 . 


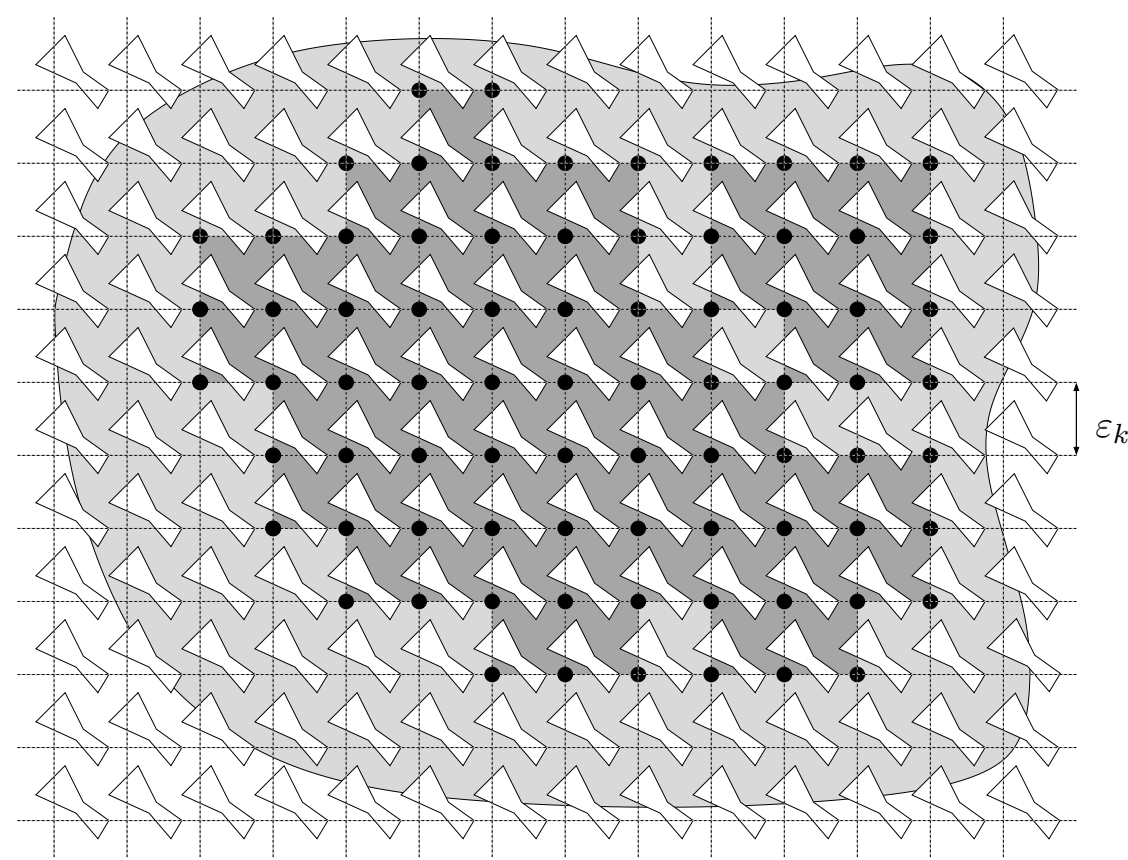

FiguRE 2. We have colored in black the balls of radius $4 r \varepsilon_{k}$ centered in the vertices of the cubes $\mathcal{Q}_{\varepsilon_{k}}^{i}, i \in H_{k}$, and in dark gray the intersection of such cubes with $\Omega_{\varepsilon_{k}}$.

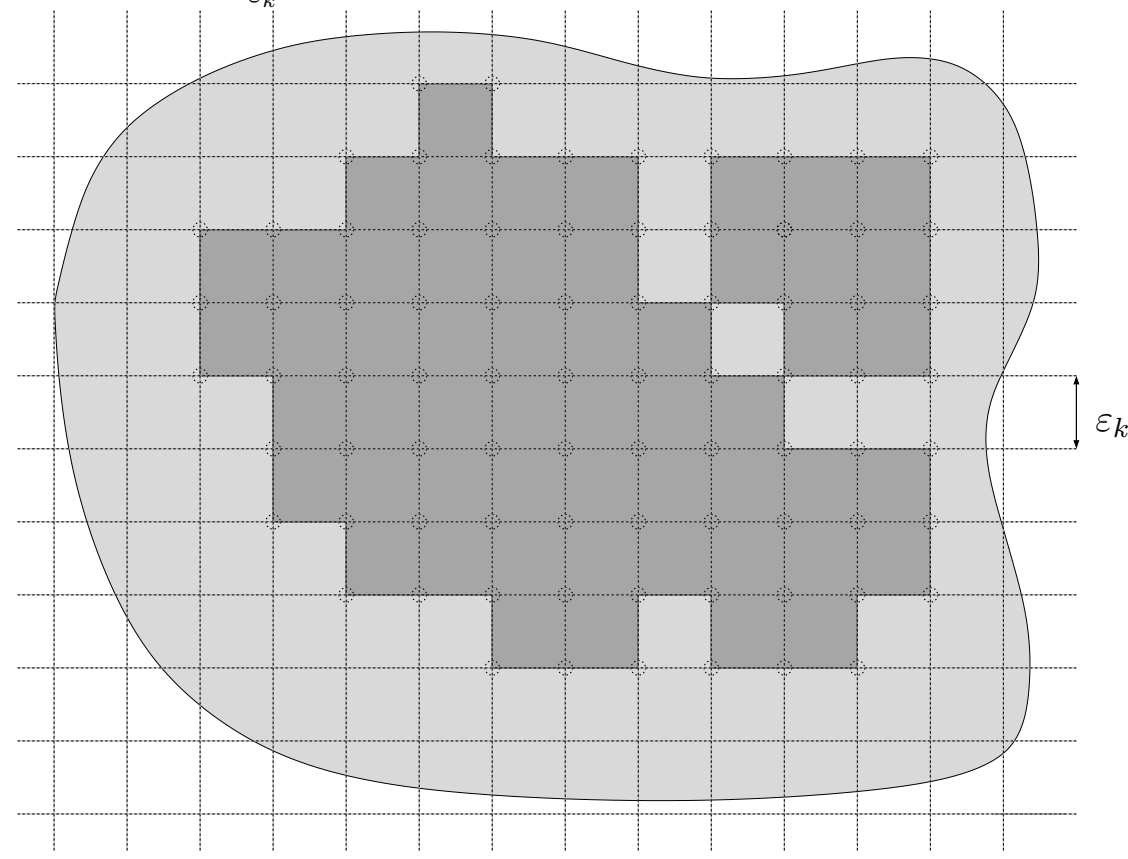

FiguRE 3. Accordingly to the previous picture, the function $v_{k}$ is obtained by applying Lemma 4 in each cube belonging to the dark gray zone and it is equal to zero in the light gray one (the remaining part of the domain $\Omega$ ). This last part vanishes as $\varepsilon_{k} \rightarrow 0^{+}$. 
Given any open set $A \subset \Omega$ we claim that $\left(v_{k}\right)$ is bounded in $G S B V^{p} \cap L^{p}(A)$. By applying estimate (2.11) in Lemma 3 between the balls of radius $r \varepsilon_{k}$ centered in the vertices of $\mathcal{Q}_{\varepsilon_{k}}^{i}, i \in H_{k}$, we bound the oscillation $O_{k}^{i}$ of the values of $w_{k}$ in such vertices as

$$
\left(O_{k}^{i}\right)^{p} \leq c\left(\varepsilon_{k}^{1-n} \int_{E_{\varepsilon_{k}}^{i}}\left|\nabla w_{k}\right| d x\right)^{p} \leq c \varepsilon_{k}^{p-n} \int_{E_{\varepsilon_{k}}^{i}}\left|\nabla w_{k}\right|^{p} d x .
$$

By combining such an estimate with (2.13)-(2.14) and (2.20) we get for every $i \in H_{k}$

$$
\int_{\mathcal{Q}_{\varepsilon_{k}}^{i}}\left|v_{k}\right|^{p} d x \leq c \int_{E_{\varepsilon_{k}}^{i}}\left(\left|u_{k}\right|^{p}+\left|\nabla u_{k}\right|^{p}\right) d x \text { and } \int_{\mathcal{Q}_{\varepsilon_{k}}^{i}}\left|\nabla v_{k}\right|^{p} d x \leq c \int_{E_{\varepsilon_{k}}^{i}}\left|\nabla u_{k}\right|^{p} d x
$$

Then, by summing up on $i \in H_{k}$,

$$
\int_{\Omega}\left|v_{k}\right|^{p} d x \leq c \int_{\Omega}\left(\left|u_{k}\right|^{p}+\left|\nabla u_{k}\right|^{p}\right) d x \text { and } \int_{\Omega}\left|\nabla v_{k}\right|^{p} d x \leq c \int_{\Omega}\left|\nabla u_{k}\right|^{p} d x .
$$

Moreover, if $k$ is large enough the only discontinuities of $v_{k}$ in $A$ are along the $(n-1)$ dimensional faces of the cubes $\mathcal{Q}_{\varepsilon_{k}}^{i}$ for $i \in I_{k} \backslash H_{k}$. Then,

$$
\mathcal{H}^{n-1}\left(S_{v_{k}} \cap A\right) \leq c \mathcal{H}^{n-1}\left(S_{u_{k}} \cap \Omega_{\varepsilon_{k}}\right) .
$$

Hence, by (2.1), we use the $G S B V^{p}$ compactness theorem (see [5, Theorem 4.36]) to infer that $\left(v_{k}\right)$ converges in $L^{1}(A)$ (up to a subsequence not relabeled for convenience) to some $v \in G S B V^{p} \cap L^{p}(A)$, with

$$
\begin{gathered}
\int_{A}|\nabla v|^{p} d x \leq c \liminf _{k} \int_{\Omega_{\varepsilon_{k}}}\left|\nabla u_{k}\right|^{p} d x<+\infty, \\
\mathcal{H}^{n-1}\left(S_{v} \cap A\right) \leq c \liminf _{k} \mathcal{H}^{n-1}\left(S_{u_{k}} \cap \Omega_{\varepsilon_{k}}\right)<+\infty .
\end{gathered}
$$

Observing that $\tilde{w}_{k}$ and $v_{k}$ coincide (at least) on the set $\cup_{i \in H_{k}}\left(\left(B_{r}\right)_{\varepsilon_{k}}^{i} \cap \mathcal{Q}_{\varepsilon_{k}}^{i}\right)$, by Lemma 1 we have that $u=v \mathcal{L}^{n}$ a.e. in $A$. By the arbitrariness of $A$ in the inequalities above and since the constant $c$ is independent from $A$, we conclude that $u$ belongs to $G S B V^{p}(\Omega)$ and estimates (2.3) are verified.

Step 3. We eliminate the cone condition requirement on $P$.

Let $t \in(0,1)$ and let $P^{t}$ be an open, $Q$-periodic and connected set having the cone property and such that $B_{4 r} \subseteq P^{t} \subseteq P$ and $\mathcal{L}^{n}\left(Q \cap\left(P \backslash P^{t}\right)\right) \leq t$. Such a set $P^{t}$ can be assembled simply by merging open balls included in $P$ (see also Lemma 8).

Let $\tilde{u}_{k}^{t}$ be defined as in (2.2), with $P^{t}$ instead of $P$ and $\Omega_{\varepsilon}^{t}:=\Omega \cap \varepsilon P^{t}$ instead of $\Omega_{\varepsilon}$. By the previous part of the proof, we know that (up to a subsequence) $\left(\tilde{u}_{k}^{t}\right)$ converges strongly in $L^{1}(\Omega)$ to some $u \in G S B V^{p} \cap L^{p}(\Omega)$. By Lemma 1 the limit $u$, and then the subsequence, is not depending on $t$. Moreover,

$$
\int_{\Omega}\left|\tilde{u}_{k}-\tilde{u}_{k}^{t}\right| d x \leq \int_{\Omega \cap \varepsilon_{k}\left(P \backslash P^{t}\right)}\left(\left|u_{k}\right|+\left|\tilde{u}_{k}^{t}\right|\right) d x \leq c t^{\frac{p-1}{p}}\left\|u_{k}\right\|_{L^{p}\left(\Omega_{\varepsilon_{k}}\right)},
$$

with the constant $c$ independent from $t$. Being $t$ arbitrary, we deduce that $\left(\tilde{u}_{k}\right)$ converges strongly in $L^{1}(\Omega)$ to $u$. Moreover, since $\Omega_{\varepsilon_{k}}^{t} \subseteq \Omega_{\varepsilon_{k}}$, inequalities (2.3) are true in this setting, too.

Finally, the sequence $\left(\tilde{u}_{k}\right)$ is bounded in $L^{\infty}(\Omega)$ if $\sup _{k}\left\|u_{k}\right\|_{L^{\infty}\left(\Omega_{\varepsilon_{k}}\right)}<+\infty$, therefore $u \in S B V^{p} \cap L^{\infty}(\Omega)$ and $\tilde{u}_{k} \rightarrow u$ strongly in $L^{p}(\Omega)$. 
In the framework of Sobolev spaces the previous approach greatly simplifies and essentially reduces to [2, Lemma 2.3], though some changes are needed since we prove it under weaker assumptions and give a slightly different conclusion.

Theorem 2. Let $\varepsilon_{k} \rightarrow 0^{+}$and let $\left(u_{k}\right)$ be a sequence in $W^{1, p}\left(\Omega_{\varepsilon_{k}}\right)$ such that

$$
\sup _{k}\left\|u_{\varepsilon_{k}}\right\|_{W^{1, p}\left(\Omega_{\varepsilon_{k}}\right)}<+\infty .
$$

Defined $\left(\tilde{u}_{k}\right)$ as in Theorem 1, then (up to a subsequence not relabeled) $\tilde{u}_{k} \rightarrow u$ strongly in $L^{1}(\Omega)$, for some $u \in W^{1, p}(\Omega)$. Moreover, there is a constant $c=c(n, p, P)$ such that

$$
\int_{\Omega}|\nabla u|^{p} d x \leq c \liminf _{k} \int_{\Omega_{\varepsilon_{k}}}\left|\nabla u_{k}\right|^{p} d x .
$$

In addition, if $P$ has the cone property, then $\tilde{u}_{k} \rightarrow u$ strongly in $L_{l o c}^{p}(\Omega)$.

Proof. The first part of the proof can be deduced directly from Theorem 1 since the limit function $u$ belongs to $G S B V^{p} \cap L^{p}(\Omega)$ and $\mathcal{H}^{n-1}\left(S_{u}\right)=0$, it belongs to $W^{1, p}(\Omega)$.

It remains to prove that the sequence $\left(\tilde{u}_{k}\right)$ is pre-compact in $L_{l o c}^{p}(\Omega)$ when $P$ has the cone property. This can be done as in Step 1 of Theorem 1 by estimating the $L^{p}$ moduli of continuity of $\left(\zeta_{k}\right)$ on each open subset $A \subset \Omega$. In doing that we use the analogue of Proposition 5 in Sobolev spaces (see for instance [23, Lemma 1.1.11]) to infer

$$
\left\|\tau_{h}\left(\zeta_{k}\right)-\zeta_{k}\right\|_{L^{p}(A)} \leq c|h|\left\|\nabla u_{k}\right\|_{L^{p}\left(\Omega_{\varepsilon_{k}}, \mathbb{R}^{n}\right)} .
$$

Being $\left(\zeta_{k}\right)$ bounded in $L^{p}(\Omega),\left(\zeta_{k}\right)$ converges (up to subsequences) strongly in $L_{l o c}^{p}(\Omega)$ to some $u \in L^{p}(\Omega)$. In addition, by passing to the infimum limit in $(2.21)$, since $c$ is depending neither on $h$ nor on $A$, we get

$$
\left\|\tau_{h}(u)-u\right\|_{L^{p}(A)} \leq c|h| \liminf _{k}\left\|\nabla u_{k}\right\|_{L^{p}\left(\Omega_{\varepsilon_{k}}, \mathbb{R}^{n}\right)},
$$

from which we infer that $u$ is actually in $W^{1, p}(\Omega)$ with (see Proposition IX.3 [11])

$$
\|\nabla u\|_{L^{p}\left(\Omega, \mathbb{R}^{n}\right)} \leq c \liminf _{k}\left\|\nabla u_{k}\right\|_{L^{p}\left(\Omega_{\varepsilon_{k}}, \mathbb{R}^{n}\right)} .
$$

Eventually, by [23, Lemma 1.1.11] we have

$$
\begin{aligned}
\left\|\tilde{u}_{k}-\zeta_{k}\right\|_{L^{p}(A)}^{p} & \leq \sum_{i \in I_{k}} \int_{E_{\varepsilon_{k}}^{i}}\left|u_{k}-f_{\left(B_{r}\right)_{\varepsilon_{k}}^{i}} u_{k}(y) d y\right|^{p} d x \\
& \leq c \varepsilon_{k}^{p} \sum_{i \in I_{k}}\left\|\nabla u_{k}\right\|_{L^{p}\left(E_{\varepsilon_{k}}^{i}, \mathbb{R}^{n}\right)}^{p} \leq c \varepsilon_{k}^{p}\left\|\nabla u_{k}\right\|_{L^{p}\left(\Omega_{\varepsilon_{k}}, \mathbb{R}^{n}\right)}^{p},
\end{aligned}
$$

and thus we infer the convergence of $\left(\tilde{u}_{k}\right)$ to $u$ in $L_{l o c}^{p}(\Omega)$.

Corollary 1. Assume $\Omega$ Lipschitz and $P$ with the cone property. Let $\varepsilon_{k} \rightarrow 0^{+}$and let $\left(u_{k}\right) \subset W^{1, p}\left(\Omega_{\varepsilon_{k}}\right)$ be a sequence such that $\sup _{k}\left\|\nabla u_{\varepsilon_{k}}\right\|_{L^{p}\left(\Omega_{\varepsilon_{k}}, \mathbb{R}^{n}\right)}<+\infty$ and $u_{k}=u^{0}$ in $\partial \Omega \cap \partial \Omega_{\varepsilon_{k}}$ for a certain $u^{0} \in W^{1, p}(\Omega)$. Defined $\left(\tilde{u}_{k}\right)$ as in Theorem 1, then (up to a subsequence not relabeled) $\tilde{u}_{k} \rightarrow u$ strongly in $L^{p}(\Omega)$, for some $u \in W^{1, p}(\Omega)$ such that $u=u^{0}$ in $\partial \Omega$.

Proof. Without loss of generality we can assume $u^{0}=0$. Then, the extension by zero of $u_{k}$ to all $\varepsilon_{k} P$ belongs to $W^{1, p}\left(\varepsilon_{k} P\right)$. Now we argue as in [2, Lemma A.4] to show that $\left(u_{k}\right)$ is bounded in $L^{p}\left(\varepsilon_{k} P\right)$. For, let $h \in \mathbb{R}^{n}$ be such that $(h+\Omega) \cap \Omega=\varnothing$, and let $\Omega^{\prime}$ be 
a bounded, open set with $(h+\Omega) \cup \Omega \subset \Omega^{\prime}$. With the role of $\Omega, A$ in Theorem 2 played by $\Omega^{\prime}, \Omega$ here, respectively, (2.22) yields

$$
\left\|u_{k}\right\|_{L^{p}\left(\Omega_{\varepsilon_{k}}\right)} \leq\left\|\zeta_{k}-u_{k}\right\|_{L^{p}\left(\Omega_{\varepsilon_{k}}\right)}+\left\|\zeta_{k}\right\|_{L^{p}\left(\Omega_{\varepsilon_{k}}\right)} \leq c \varepsilon_{k}\left\|\nabla u_{k}\right\|_{L^{p}\left(\Omega_{\varepsilon_{k}}^{\prime}, \mathbb{R}^{n}\right)}+\left\|\zeta_{k}\right\|_{L^{p}\left(\Omega_{\varepsilon_{k}}\right)} .
$$

To bound the last term take note that $\zeta_{k}=0$ on $h+\Omega$ by the choice of $h \in \mathbb{R}^{n}$, then (2.21) implies

$$
\left\|\zeta_{k}\right\|_{L^{p}\left(\Omega_{\varepsilon_{k}}\right)}=\left\|\tau_{h}\left(\zeta_{k}\right)-\zeta_{k}\right\|_{L^{p}\left(\Omega_{\varepsilon_{k}}\right)} \leq c|h|\left\|\nabla u_{k}\right\|_{L^{p}\left(\Omega_{\varepsilon_{k}}^{\prime}, \mathbb{R}^{n}\right)} .
$$

By collecting the last two inequalities, we infer that $\left(u_{k}\right)$ is bounded in $L^{p}\left(\Omega_{\varepsilon_{k}}\right)$ and then in $L^{p}\left(\varepsilon_{k} P\right)$. By applying Theorem 2 in some open and bounded set compactly containing $\Omega^{\prime}$, we obtain that up to a subsequence $\left(\tilde{u}_{k}\right)$ converges strongly in $L^{p}\left(\Omega^{\prime}\right)$ to some $u \in W^{1, p}\left(\Omega^{\prime}\right)$. Since $\tilde{u}_{k}=0$ in $\Omega_{\varepsilon_{k}}^{\prime} \backslash \Omega$, by Lemma 1 it follows that $u=0$ in $\Omega^{\prime} \backslash \Omega$.

\section{LUSIN TYPE APPROXIMATION}

In order to identify the volume energy density of the $\Gamma$-limit in the homogenization result, we decompose a given sequence in $S B V^{p}\left(\Omega_{\varepsilon}\right)$ in the sum of a function in $S B V^{p}(\Omega)$ and a sequence in $W^{1, \infty}\left(\mathbb{R}^{n}\right)$ keeping the energy bounds on $\Omega_{\varepsilon}$ and the same $L^{1}(\Omega)$ limit. To do that we follow a by now classical strategy based on maximal function estimates and Lipschitz extension techniques developed in [3]. A similar approach has been used in [18, Step 2 of Proposition 5.3] in the setting of perforated domains. Despite the result there is correct, a technical difficulty has been overlooked. The argument in [18, Step 2 of Proposition 5.3] hinges on [21, Lemma 2.1], in which the same result for the case of nonperforated domains, i.e., sequences in $S B V(\Omega)$, had been previously shown. That proof contains an inaccuracy which can be easily fixed when invoking the use of [17, Theorem 2 in Section 6.6.2]. Indeed, the maximal function operator employed in [21, Lemma 2.1] is not clearly defined, it can be interpreted either to be the one related to the zero extension of the total variation measure outside $\Omega$, or to be the one related to the zero extension of the given function out of $\Omega$. In both cases the result by [17] can not be directly applied, though only simple changes have to be performed to conclude the proof. For instance, an extension of the functions to all $\mathbb{R}^{n}$ maintaining the vanishing condition of the measure of their jump sets suffices (see Step 1 of Proposition 3).

As a consequence, a constant that in [21, Lemma 2.1] depends only on the space dimension $n$, actually turns out to depend also on the geometry of the reference domain $\Omega$. Clearly, this fact prevents the straightforward application of [21, Lemma 2.1] in the framework of varying domains $\Omega_{\varepsilon}$ as done in [18, Step 2 of Proposition 5.3], and requires a control of the behavior of those constants as $\varepsilon \rightarrow 0^{+}$.

In the sequel, we clarify the stage providing full proofs, though some arguments are well known in literature.

First, let us fix some notations. Given a positive and finite measure Radon $\mu$ in $\mathbb{R}^{n}$, we denote by $M(\mu)$ the maximal function of $\mu$ :

$$
M(\mu)(x):=\sup _{r>0} \frac{\mu\left(B_{r}(x)\right)}{\mathcal{L}^{n}\left(B_{r}(x)\right)} .
$$

We simply write $M(w)$ when $\mu=w \mathcal{L}^{n}$ for some $w \in L^{1}\left(\mathbb{R}^{n}\right)$.

If $A$ is an open set, the zero extension of the measure $\mu$ out of $A$ is denoted by $\mu \chi_{A}$.

Lemma 5. Let $R$ be a open rectangle in $\mathbb{R}^{n}$ and let $\phi: R \rightarrow A$ be a bi-Lipschitz transformation. Then, there exists a positive constant $c$ depending on $n$ and on the product of the 
Lipschitz constants of $\phi$ and $\phi^{-1}$, such that for every $u \in B V(A)$ it holds

$$
|u(x)-u(y)| \leq c|x-y|\left[M\left(|D u| \chi_{A}\right)(x)+M\left(|D u| \chi_{A}\right)(y)\right]
$$

for $\mathcal{L}^{n}$ a.e. $x, y \in A$.

Proof. Step 1. Assume initially that $\phi$ is the identity, that is $R=A$.

Let $r>0$ and let $x \in A$ be a Lebesgue point for $u$. By Proposition 4,

$$
\int_{B_{r}(x) \cap A}\left|u(z)-u_{B_{r}(x) \cap A}\right| d z \leq c(n) r|D u|\left(B_{r}(x) \cap A\right) .
$$

By the definition of $M\left(|D u| \chi_{A}\right)$, then it follows that

$$
\frac{1}{\mathcal{L}^{n}\left(B_{r}(x)\right)} \int_{B_{r}(x) \cap A}\left|u(z)-u_{B_{r}(x) \cap A}\right| d z \leq c(n) r M\left(|D u| \chi_{A}\right)(x) .
$$

Since $A$ is a rectangle, $\mathcal{L}^{n}\left(B_{\frac{r}{2^{k+1}}}(x) \cap A\right) \geq c(n) \mathcal{L}^{n}\left(B_{\frac{r}{2^{k}}}(x)\right)$. Therefore

$$
\begin{aligned}
& \left|u_{B \frac{r}{2^{k}}}(x) \cap A-u_{B_{\frac{2^{k}}{k^{* 1}}}}(x) \cap A\right| \leq f_{B \frac{r}{2^{k+1}}(x) \cap A}\left|u(z)-u_{B \frac{r}{2^{k}}}(x) \cap A\right| d z \\
& \quad \leq \frac{c(n)}{\mathcal{L}^{n}\left(B_{\frac{r}{2^{k}}}(x)\right)} \int_{B_{\frac{r}{2^{k}}}(x) \cap A}\left|u(z)-u_{B_{\frac{r}{2^{k}}}}(x) \cap A\right| d z \leq \frac{c(n) r}{2^{k}} M\left(|D u| \chi_{A}\right)(x) .
\end{aligned}
$$

Since $x$ is a Lebesgue point, we have

$$
u(x)=\lim _{r \rightarrow 0^{+}} u_{B_{r}(x)}=\lim _{k \rightarrow \infty} u_{B_{\frac{r}{2^{k}}}}(x)
$$

and then

$$
\left|u(x)-u_{B_{r}(x) \cap A}\right|=\sum_{k=0}^{\infty}\left|u_{B \frac{r}{2^{k}}}(x) \cap A-u_{B \frac{r}{2^{k+1}}}(x) \cap A\right| \leq c(n) r M\left(|D u| \chi_{A}\right)(x) .
$$

Consider now two distinct Lebesgue points $x, y \in A$ and set $r=|x-y|$. Since $A$ is a rectangle, $\mathcal{L}^{n}\left(B_{r}(x) \cap B_{r}(y) \cap A\right) \geq c(n) \mathcal{L}^{n}\left(B_{r}(x)\right)$. Therefore

$$
\begin{aligned}
& \left|u_{B_{r}(x) \cap A}-u_{B_{r}(y) \cap A}\right| \leq f_{B_{r}(x) \cap B_{r}(y) \cap A}\left(\left|u(z)-u_{B_{r}(x) \cap A}\right|+\left|u(z)-u_{B_{r}(y) \cap A}\right|\right) d z \\
& \quad \leq \frac{c(n)}{\mathcal{L}^{n}\left(B_{r}(x)\right)} \int_{B_{r}(x) \cap A}\left|u-u_{B_{r}(x) \cap A}\right| d z+\frac{c(n)}{\mathcal{L}^{n}\left(B_{r}(y)\right)} \int_{B_{r}(y) \cap A}\left|u-u_{B_{r}(y) \cap A}\right| d z \\
& \quad \leq c(n) r\left[M\left(|D u| \chi_{A}\right)(x)+\left(M\left(|D u| \chi_{A}\right)(y)\right] .\right.
\end{aligned}
$$

Gathering (3.2) and (3.3) through the triangular inequality we get (3.1).

Step 2. In order to raise the result obtained in the previous step to the general case, we consider the pullback $w(z):=u(\phi(z))$. Denote by $l$ and $l^{\prime}$ the Lipschitz constants of $\phi$ and $\phi^{-1}$, respectively. By [5, Theorem 3.16] $w$ belongs to $B V(R)$ with

$$
|D w|(U) \leq\left(l^{\prime}\right)^{n-1} \phi_{\#}^{-1}|D u|(U)
$$

for any Borel set $U \subseteq R$, where $\phi_{\#}^{-1}|D u|$ is the push-forward of the measure $|D u|$ through $\phi^{-1}$. Since $\phi\left(B_{r}(z)\right) \subseteq B_{r l}(\phi(z))$, by (3.4) it follows

$$
|D w|\left(B_{r}(z) \cap R\right) \leq\left(l^{\prime}\right)^{n-1}|D u|\left(\phi\left(B_{r}(z) \cap R\right)\right) \leq\left(l^{\prime}\right)^{n-1}|D u|\left(B_{r l}(\phi(z)) \cap A\right),
$$


and thus, by taking into account that $\phi^{-1}\left(B_{r / l^{\prime}}(\phi(z))\right) \subseteq B_{r}(z)$, a change of variables implies

$$
M\left(|D w| \chi_{R}\right)(z) \leq l^{2 n}\left(l^{\prime}\right)^{2 n-1} M\left(|D u| \chi_{A}\right)(\phi(z)) .
$$

Finally, by Step 1 and since $\phi$ enjoys the Lusin (N) property, we infer for $\mathcal{L}^{n}$ a.e. $x, y \in A$

$$
\begin{aligned}
|u(x)-u(y)| & =\left|w\left(\phi^{-1}(x)\right)-w\left(\phi^{-1}(y)\right)\right| \\
& \leq c(n)\left|\phi^{-1}(x)-\phi^{-1}(y)\right|\left[M\left(|D w| \chi_{R}\right)\left(\phi^{-1}(x)\right)+M\left(|D w| \chi_{R}\right)\left(\phi^{-1}(y)\right)\right] \\
& \leq c(n)\left(l l^{\prime}\right)^{2 n}|x-y|\left[M\left(|D u| \chi_{A}\right)(x)+\left(M\left(|D u| \chi_{A}\right)(y)\right] .\right.
\end{aligned}
$$

The result in the sequel is instrumental in order to apply the previous lemma in our framework.

Lemma 6. Assume that $P$ is Lipschitz regular. Given $x, y \in P$, there exist an open rectangle $R$, an open set $A \subseteq P$ containing $x$ and $y$, and a bi-Lipschitz transformation $\phi: R \rightarrow A$ such that both $\phi$ and $\phi^{-1}$ have Lipschitz constants dominated by a constant $c=c(n, P)$.

Proof. For each $x \in \overline{P \cap Q}$ we choose an open rectangle $R_{r}(x)$, centered in $x$ and with diameter $r=r(x)$, as follows

- if $x \in P$, we require only that $R_{r}(x) \subseteq P$;

- if $x \in \partial P$, then we choose $R_{r}(x)$ in such a way that there exist a Lipschitz function $f_{x}:(0,1)^{n-1} \rightarrow(0,1)$ and an affine transformation $\varphi_{x}: Q \rightarrow R_{r}(x)$ such that, writing $z \in Q$ as $\left(z^{\prime}, z_{n}\right) \in(0,1)^{n-1} \times(0,1)$,

$$
R_{r}(x) \cap P=\varphi_{x}\left(\left\{z \in Q: z_{n}<f_{x}\left(z^{\prime}\right)\right\}\right) .
$$

This is possible since $P$ is a Lipschitz domain.

Now we extract from $\left\{R_{r}(x): x \in \overline{P \cap Q}\right\}$ a finite subcovering $\left\{R_{r}\left(x_{k}\right): k=1, \ldots, m\right\}$ of $\overline{P \cap Q}$. Let $\delta>0$ be such that, if $x \in \overline{P \cap Q}$, then $B_{\delta}(x) \subseteq R_{r}\left(x_{k}\right)$ for some $k \in$ $\{1, \ldots, m\}$. Thank to the periodicity of $P$, the thesis is right away proved when $|x-y|<\delta$.

Instead, if $|x-y| \geq \delta$ we proceed as follow. As before, for each $x \in \overline{P \cap Q}$ we choose an open rectangle $R_{r}(x)$, this time with the additional constraint $r<\delta / 2$, and extract a finite subcovering $\left\{R_{r}\left(x_{k}\right): k=1, \ldots, m^{\prime}\right\}$ of $\overline{P \cap Q}$.

Let $k, j \in\left\{1, \ldots, m^{\prime}\right\}$ and $i, j \in \mathbb{Z}^{n}$ such that $x \in R_{r}\left(x_{k}+i\right)$ and $y \in R_{r}\left(x_{j}+h\right)$. To complete the proof it is sufficient to find an open rectangle $R \subseteq \mathbb{R}^{n}$, an open set $A \subseteq P$ including $P \cap R_{r}\left(x_{k}+i\right)$ and $P \cap R_{r}\left(x_{j}+h\right)$, and a bi-Lipschitz application $\phi: R \rightarrow A$ in such a way that the Lipschitz constants of $\phi$ and $\phi^{-1}$ do not depend on $i$ and $h$. By translating and rotating the coordinate system, we can assume $i=0$ and $h=\left(h_{1}, \ldots, h_{n}\right) \in \mathbb{N}^{n}$.

We examine first the case $h=0$. Since the constraint $r<\delta / 2$ implies that $R_{r}\left(x_{k}\right)$ and $R_{r}\left(x_{j}\right)$ are well separated, we can build an open set $U \subseteq \mathbb{R}^{n}$ and a bi-Lipschitz application $\psi:(0,1)^{n-1} \times(-2,1) \rightarrow U$ such that $\psi\left((0,1)^{n-1} \times[-1,0]\right) \subseteq P$, and $\left.\psi\right|_{(0,1)^{n-1} \times(-2,-1)}$ and $\left.\psi\right|_{(0,1)^{n-1} \times(0,1)}$ are affine transformations on $R_{r}\left(x_{k}\right)$ and $R_{r}\left(x_{j}\right)$, respectively. Setting $A:=P \cap U$, we define $\phi$ according to the following four cases:

- $x_{k}, x_{j} \in P$. Since $R_{r}\left(x_{k}\right), R_{r}\left(x_{j}\right) \subseteq P$, we take $\phi=\psi$; 
- $x_{k} \in P$ and $x_{j} \in \partial P$. If $n \geq 3$, by rotating $f_{x_{j}}$ around the axis $z_{n}=1 / 2$, we can assume that $\varphi_{x_{j}}=\left.\psi\right|_{(0,1)^{n-1} \times(0,1)}$ (this is always the case if $\left.n=2\right)$. Then, the map

$$
\phi(z):= \begin{cases}\psi(z) & \text { if } z \in(0,1)^{n-1} \times(-2,0], \\ \psi\left(\left(z^{\prime}, f_{x_{j}}\left(z^{\prime}\right) z_{n}\right)\right) & \text { if } z \in(0,1)^{n-1} \times(0,1),\end{cases}
$$

is what we are looking for (see Figure 4);

- $x_{k} \in \partial P$ and $x_{j} \in P$, or $x_{k}, x_{j} \in \partial P$. We argue similarly to the previous case.

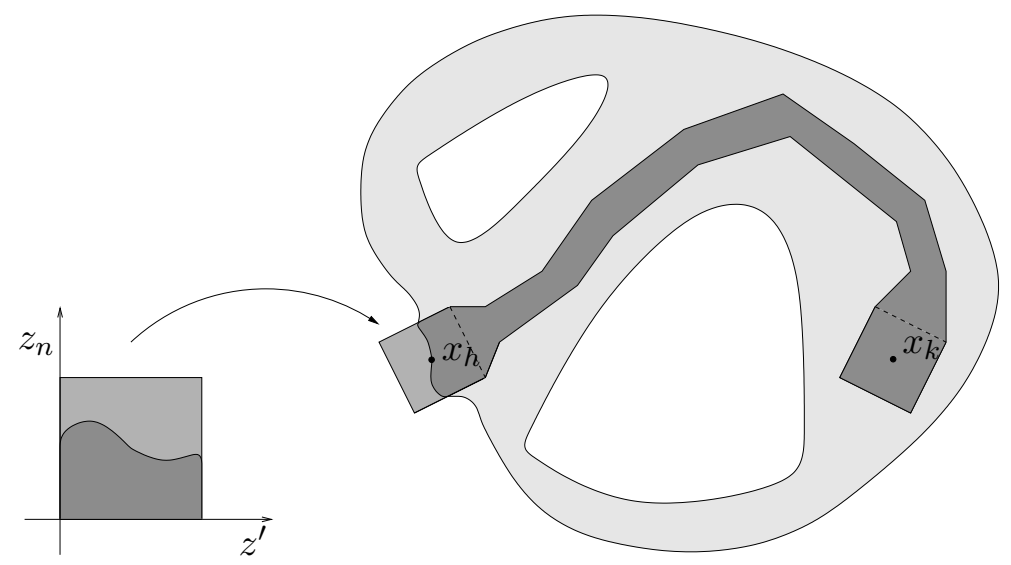

FIGURE 4

To conclude we examine the case $|h|:=h_{1}+\ldots+h_{n} \geq 1$. We remark that the construction of the set $U$ cannot be made arbitrarily since we need to control the Lipschitz constants of $\phi$ and $\phi^{-1}$.

Let $\mathrm{e}_{1}, \ldots, \mathrm{e}_{n}$ be the unit vectors of the canonical base $\mathbb{E}$ of $\mathbb{R}^{n}$, and let $R_{r}\left(x_{s}\right) \subseteq P \cap Q$ be an open rectangle, not necessarily in the extracted subcovering, well separated from both $R_{r}\left(x_{k}\right)$ and $R_{r}\left(x_{j}\right)$. We write

$$
\begin{aligned}
& y_{-1}=x_{k}, y_{0}=x_{s}, y_{1}=x_{s}+\mathrm{e}_{1}, \ldots, y_{h_{1}}=x_{s}+h_{1} \mathrm{e}_{1}, y_{h_{1}+1}=x_{s}+h_{1} \mathrm{e}_{1}+\mathrm{e}_{2}, \ldots, \\
& y_{h_{1}+h_{2}}=x_{s}+h_{1} \mathrm{e}_{1}+h_{2} \mathrm{e}_{2}, \ldots, y_{|h|}=x_{s}+h, y_{|h|+1}=x_{j}+h .
\end{aligned}
$$

We can build an open set $U \subseteq \mathbb{R}^{n}$ (see Figure 5) and a bi-Lipschitz application $\psi$ : $(0,1)^{n-1} \times(-2,2|h|+3) \rightarrow U$ such that

- $\psi\left((0,1)^{n-1} \times[-1,2|h|+2]\right) \subseteq P$;

- $\left.\psi\right|_{(0,1)^{n-1} \times(2 t, 2 t+3)}$ joins $R_{r}\left(y_{t}\right)$ with $R_{r}\left(y_{t+1}\right)$ for $t=-1, \ldots,|h|$ (this is possible thanks to the choice of $x_{s}$ since the relevant rectangles are well separated);

- $\left.\psi\right|_{(0,1)^{n-1} \times(-2,-1)}$ (resp., $\left.\left.\psi\right|_{(0,1)^{n-1} \times(2|h|+2,2|h|+3)}\right)$ is an affine transformation onto $R_{r}\left(x_{k}\right)$ (resp., $\left.R_{r}\left(x_{j}+h\right)\right)$;

- $\left.\psi\right|_{(0,1)^{n-1} \times(2 t, 2 t+2)}=\tau_{2 t \mathrm{e}_{1}}\left(\left.\psi\right|_{(0,1)^{n-1} \times(0,2)}\right)$, for $t=1, \ldots, h_{1}-1$, we argue similarly on the branches in the remaining directions $\mathrm{e}_{2}, \ldots, \mathrm{e}_{n}$.

The last (periodicity) condition ensures that the Lipschitz constants of $\psi$ and $\psi^{-1}$ are not depending on how large $h_{1}, \ldots, h_{n}$ are.

Eventually, we set $A:=P \cap U$ and conveniently modify $\psi$ on $(0,1)^{n-1} \times(-2,-1)$ and on $(0,1)^{n-1} \times(2|h|+2,2|h|+3)$ arguing as in the case $i=h=0$ in order to get $\phi$. 


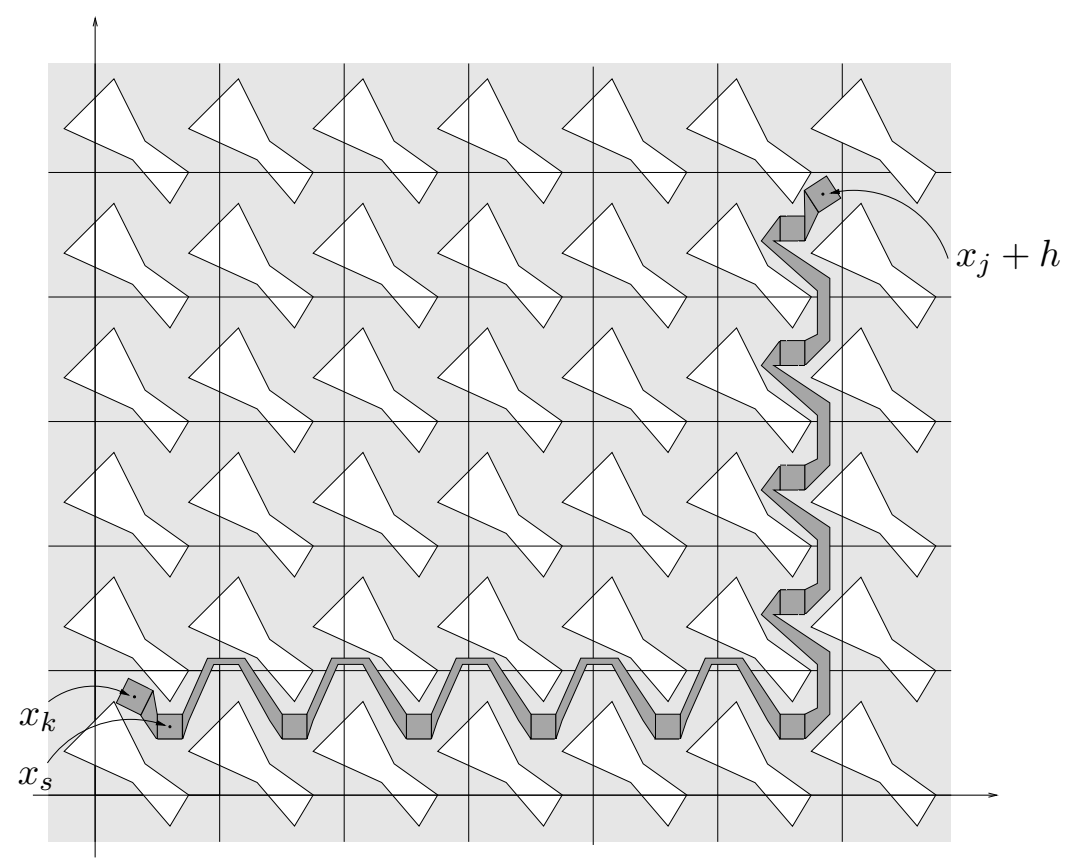

FigURE 5

Remark 2. In Lemma 6 the constant $c$ is invariant by rescaling: $c(n, P)=c(n, \varepsilon P)$ for any $\varepsilon>0$.

The following estimate of the oscillation of a function $u \in S B V(\varepsilon P)$ in terms of the maximal function of $D u$ is the key of our approximation result.

Theorem 3. Assume that $P$ is Lipschitz regular, there exists a constant $c=c(n, P)$ with the following property: for each $\varepsilon>0$ and for each $u \in S B V(\varepsilon P)$,

$$
|u(x)-u(y)| \leq c|x-y|\left[M\left(|D u| \chi_{\varepsilon P}\right)(x)+M\left(|D u| \chi_{\varepsilon P}\right)(y)\right]
$$

for $\mathcal{L}^{n}$ a.e. $x, y \in \varepsilon P$.

Proof. It is a straightforward consequence of Lemma 5, Lemma 6 and Remark 2.

We are now ready to prove a decomposition result for sequence in $S B V$ in the framework of periodically perforated domains. In doing that we have been inspired by [20, Theorem 6.3], [5, Theorem 5.36] and [19, Lemma 1.2].

Proposition 3. Assume that $P$ is Lipschitz regular. Let $\varepsilon_{k} \rightarrow 0^{+}$and let $\left(u_{k}\right)$ be a sequence in $L^{1}(\Omega)$ such that $u_{k} \rightarrow u$ strongly in $L^{1}(\Omega)$ to some $u \in S B V^{p}(\Omega),\left.u_{k}\right|_{\Omega_{\varepsilon_{k}}} \in$ $S B V^{p}\left(\Omega_{\varepsilon_{k}}\right)$ and

$$
\sup _{k}\left(\int_{\Omega_{\varepsilon_{k}}}\left|\nabla u_{k}\right|^{p} d x+\mathcal{H}^{n-1}\left(S_{u_{k}} \cap \Omega_{\varepsilon_{k}}\right)\right)<+\infty .
$$

Then, there exist a subsequence $k_{j} \rightarrow+\infty$ and a sequence $\left(w_{j}\right) \subset W^{1, \infty}(\Omega)$ such that $w_{j} \rightarrow 0$ strongly in $L^{\infty}(\Omega), w_{j}=0$ on a neighborhood of $\partial \Omega,\left(\chi_{\Omega_{\varepsilon_{k_{j}}}} \nabla w_{j}\right)$ is p-equiintegrable and

$$
\lim _{j} \mathcal{L}^{n}\left(\left\{x \in \Omega_{\varepsilon_{k_{j}}}: u_{k_{j}}(x) \neq u(x)+w_{j}(x)\right\}\right)=0 .
$$


Proof. We divide the proof into several steps.

Step 1. By truncating $\left(u_{k}-u\right)$ we find $\left(\vartheta_{k}\right) \subseteq L^{1}\left(\mathbb{R}^{n}\right) \cap S B V^{p}\left(\varepsilon_{k} P\right)$ with gradients bounded in $L^{p}\left(\varepsilon_{k} P, \mathbb{R}^{n}\right)$, with singular part of the distributional derivative vanishing on $\varepsilon_{k} P$, converging to 0 in $L^{\infty}\left(\mathbb{R}^{n}\right)$ and satisfying (3.7).

For each $\delta>0$ consider the function $u_{k, \delta}:=\left(u_{k}-u\right) \vee(-\delta) \wedge \delta$. Noting that $u_{k, \delta}(x)=$ $u_{k}(x)-u(x)$ if $\left|u_{k}(x)-u(x)\right| \leq \delta$ we have

$$
\mathcal{L}^{n}\left(\left\{x \in \Omega: u_{k, \delta}(x) \neq u_{k}(x)-u(x)\right\}\right) \leq \frac{1}{\delta} \int_{\Omega}\left|u_{k}-u\right| d x .
$$

Since $u_{k} \rightarrow u$ strongly in $L^{1}(\Omega)$, we can choose $\delta_{k} \rightarrow 0^{+}$such that the function $\zeta_{k}:=u_{k, \delta_{k}}$ satisfies $\lim _{k} \mathcal{L}^{n}\left(\left\{x \in \Omega: \zeta_{k}(x) \neq u_{k}(x)-u(x)\right\}\right)=0$. Clearly, $\zeta_{k} \rightarrow 0$ strongly in $L^{\infty}(\Omega)$ since $\left\|\zeta_{k}\right\|_{L^{\infty}(\Omega)} \leq \delta_{k}$, and moreover $\left.\zeta_{k}\right|_{\Omega_{\varepsilon_{k}}} \in S B V^{p}\left(\Omega_{\varepsilon_{k}}\right)$ with

$$
\int_{\Omega_{\varepsilon_{k}}}\left|\nabla \zeta_{k}\right|^{p} d x \leq \int_{\Omega_{\varepsilon_{k}}}\left|\nabla\left(u_{k}-u\right)\right|^{p} d x, \quad S_{\zeta_{k}} \subseteq S_{u_{k}} \cup S_{u} .
$$

Finally, (3.6) yields

$$
\lim _{k}\left|D^{s} \zeta_{k}\right|\left(\Omega_{\varepsilon_{k}}\right) \leq \lim _{k} \delta_{k} \mathcal{H}^{n-1}\left(\left(S_{u_{k}} \cup S_{u}\right) \cap \Omega_{\varepsilon_{k}}\right)=0 .
$$

We extend $\zeta_{k}$ out of $\Omega$ via a cut-off argument. To this aim for each $\eta>0$ consider an open set $\Omega^{\eta} \lessdot \Omega$ in such a way that $\mathcal{L}^{n}\left(\Omega \backslash \Omega^{\eta}\right) \rightarrow 0$ as $\eta \rightarrow 0^{+}$, and a function $\varphi_{\eta} \in C_{c}^{1}(\Omega,[0,1])$ such that $\varphi_{\eta}=1$ in $\Omega^{\eta}$. Let $\zeta_{k, \eta}:=\varphi_{\eta} \zeta_{k}$, then $S_{\zeta_{k, \eta}} \subseteq S_{\zeta_{k}}$ and

$$
\int_{\Omega_{\varepsilon_{k}}}\left|\nabla \zeta_{k, \eta}\right|^{p} d x \leq 2^{p-1}\left(\int_{\Omega_{\varepsilon_{k}}}\left|\nabla \zeta_{k}\right|^{p} d x+\mathcal{L}^{n}(\Omega)\left\|\nabla \varphi_{\eta}\right\|_{L^{\infty}\left(\Omega, \mathbb{R}^{n}\right)}^{p} \delta_{k}^{p}\right) .
$$

As $\zeta_{k} \rightarrow 0$ strongly in $L^{\infty}(\Omega)$ we can choose $\eta_{k} \rightarrow 0^{+}$such that the functions defined by $\vartheta_{k}:=\zeta_{k, \eta_{k}}$ in $\Omega$ and $\vartheta_{k}:=0$ in $\mathbb{R}^{n} \backslash \Omega$ belong to $S B V^{p}\left(\varepsilon_{k} P\right)$, with

$$
\sup _{j} \int_{\varepsilon_{k} P}\left|\nabla \vartheta_{k}\right|^{p} d x<+\infty .
$$

Furthermore, $\left(\vartheta_{k}\right)$ satisfies by construction $\lim _{k} \mathcal{L}^{n}\left(\left\{x \in \Omega: \zeta_{k}(x) \neq \vartheta_{k}(x)\right\}\right)=0$ and $\lim _{k}\left|D^{s} \vartheta_{k}\right|\left(\varepsilon_{k} P\right)=0$.

Step 2. We truncate $\vartheta_{k}$ via the maximal function, getting a sequence $\left(v_{k}\right) \subseteq W^{1, \infty}\left(\mathbb{R}^{n}\right)$ with gradients bounded in $L^{p}\left(\varepsilon_{k} P, \mathbb{R}^{n}\right)$ and satisfying (3.7).

Let $\lambda_{k}:=\left|D^{s} \vartheta_{k}\right|^{\frac{1}{1-p}}\left(\varepsilon_{k} P\right)$, then $\lambda_{k} \rightarrow+\infty$ as $k \rightarrow+\infty$. Inequality (3.5) shows that the restriction of $\vartheta_{k}$ to $U_{k}:=\left\{x \in \varepsilon_{k} P: M\left(\left|D \vartheta_{k}\right| \chi_{\varepsilon_{k} P}\right)(x) \leq \lambda_{k}\right\}$ is a Lipschitz function with Lipschitz constant dominated from above by $2 c(n, P) \lambda_{k}$. Let $v_{k}$ be an extension of $\left.\vartheta_{k}\right|_{U_{k}}$ to $\mathbb{R}^{n}$ with the same Lipschitz constant. Chebyshev inequality and $[24$, Theorem $1(\mathrm{c})$ ] give for every $\lambda>0$

$$
\begin{aligned}
\mathcal{L}^{n}\left(\left\{x \in \varepsilon_{k} P: M\left(\mu_{k}\right)(x)>\lambda\right\}\right) & \leq \frac{1}{\lambda^{p}} \int_{\left\{x \in \varepsilon_{k} P: M\left(\mu_{k}\right)(x)>\lambda\right\}} M^{p}\left(\mu_{k}\right)(x) d x \\
& \leq \frac{c(n, p)}{\lambda^{p}} \int_{\left\{x \in \varepsilon_{k} P:\left|\nabla \vartheta_{k}\right|(x)>\lambda\right\}}\left|\nabla \vartheta_{k}\right|^{p}(x) d x
\end{aligned}
$$


where we have used the short hand notation $\mu_{k}=\left|\nabla \vartheta_{k}\right| \chi_{\varepsilon_{k} P}$. Moreover, a Besicovitch type covering argument gives

$$
\mathcal{L}^{n}\left(\left\{x \in \varepsilon_{k} P: M\left(\left|D^{s} \vartheta_{k}\right| \chi_{\varepsilon_{k} P}\right)(x)>\lambda\right\}\right) \leq \frac{c(n)}{\lambda}\left|D^{s} \vartheta_{k}\right|\left(\varepsilon_{k} P\right) .
$$

Equality $\left|D \vartheta_{k}\right|=\left|\nabla \vartheta_{k}\right| \mathcal{L}^{n}+\left|D^{s} \vartheta_{k}\right|$ yields that the set $\left(\varepsilon_{k} P\right) \backslash U_{k}$ is included in

$$
\left\{x \in \varepsilon_{k} P: M\left(\left|\nabla \vartheta_{k}\right| \chi_{\varepsilon_{k} P}\right)(x)>\lambda_{k} / 2\right\} \cup\left\{x \in \varepsilon_{k} P: M\left(\left|D^{s} \vartheta_{k}\right| \chi_{\varepsilon_{k} P}\right)(x)>\lambda_{k} / 2\right\},
$$

thus gathering (3.8) and (3.9) with $\lambda=\lambda_{k} / 2$ we get $\lim _{k} \mathcal{L}^{n}\left(\left(\varepsilon_{k} P\right) \backslash U_{k}\right)=0$ and

$$
\begin{aligned}
\sup _{k} \int_{\varepsilon_{k} P}\left|\nabla v_{k}\right|^{p} d x & \leq\left[\sup _{k} \int_{\varepsilon_{k} P}\left|\nabla \vartheta_{k}\right|^{p} d x+\left(2 c(n, P) \lambda_{k}\right)^{p} \mathcal{L}^{n}\left(\left(\varepsilon_{k} P\right) \backslash U_{k}\right)\right] \\
& \leq(1+c(n, p, P)) \sup _{k} \int_{\varepsilon_{k} P}\left|\nabla \vartheta_{k}\right|^{p} d x+c(n, p, P)<+\infty .
\end{aligned}
$$

Step 3. We truncate suitably $\left(v_{k}\right)$ to get a $p$-equiintegrable (sub)sequence on $\Omega_{\varepsilon_{k}}$ satisfying (3.7).

By using again [24, Theorem 1(c)], from estimate (3.10) we infer that the sequence $l_{k}:=M_{k}^{p}\left(\left|\nabla v_{k}\right| \chi_{\varepsilon_{k} P}\right)$ is bounded in $L^{1}\left(\mathbb{R}^{n}\right)$. Therefore, by Lemma 7 , there exists a subsequence $\left(l_{k_{j}}\right)$ such that $\left(l_{k_{j}} \wedge j^{p}\right)$ is equiintegrable.

Arguing as in the previous step, we can find a Lipschitz function $w_{j}$ coinciding with $v_{k_{j}}$ on $V_{j}:=\left\{x \in \varepsilon_{k_{j}} P: M\left(\left|\nabla v_{k_{j}}\right| \chi_{\varepsilon_{k_{j}} P}\right)(x) \leq j\right\}$ and having Lipschitz constant smaller than $2 c(n, P) j$. Moreover, $\lim _{j} \mathcal{L}^{n}\left(\left(\varepsilon_{k_{j}} P\right) \backslash V_{j}\right)=0$ and therefore

$$
\lim _{j} \mathcal{L}^{n}\left(\left\{x \in \varepsilon_{k_{j}} P: v_{k_{j}}(x) \neq w_{j}(x)\right\}\right) \leq \mathcal{L}^{n}\left(\left(\varepsilon_{k_{j}} P\right) \backslash\left(U_{k_{j}} \cup V_{j}\right)\right)=0 .
$$

In addition, for $\mathcal{L}^{n}$ a.e. $x \in V_{j}$ we have

$$
\left|\nabla w_{j}(x)\right|=\left|\nabla v_{k_{j}}(x)\right| \leq M\left(\left|\nabla v_{k_{j}}\right| \chi_{\varepsilon_{k_{j}} P}\right)(x)=M\left(\left|\nabla v_{k_{j}}\right| \chi_{\varepsilon_{k_{j}} P}\right)(x) \wedge j,
$$

and for $\mathcal{L}^{n}$ a.e. $x \in\left(\varepsilon_{k_{j}} P\right) \backslash V_{j}$

$$
\left|\nabla w_{j}(x)\right| \leq c(n, P) j=c(n, P)\left[M\left(\left|\nabla v_{k_{j}}\right| \chi_{\varepsilon_{k_{j}}} P\right)(x) \wedge j\right],
$$

from which we conclude that $\left|\nabla w_{j}\right| \chi_{\varepsilon_{k_{j}} P}$ is $p$-equiintegrable.

Eventually, we truncate $w_{j}$ between $\delta_{k_{j}}$ and $-\delta_{k_{j}}$ and apply again the cut-off procedure performed in Step 1 in order to get a sequence satisfying all the required properties.

\section{Homogenization}

We give an application of the results in the previous sections to prove the homogenization of non equi-coercive energies in $S B V^{p}$ and in $W^{1, p}$.

We follow the approach by [18], extending the results there to the case in which $P$ is only assumed connected (see also [14]). A major technical difference is that we avoid the use of any extension result in Sobolev spaces on perforated domains, which was an instrumental tool to identify the density $f_{\text {hom }}$ in [18, Proposition 5.1]. Thus, in the sequel we indicate how the arguments of [18] must be appropriately restated and provide full proofs only when our strategy departs from the original one.

Furthermore, as a consequence of this, we are able to give a new proof of the classical homogenization theorem for degenerate energies on $W^{1, p}$, too. 
Let us first set some notation. Given $\varepsilon>0$, let $W_{p e r}^{1, p}(Q \cap \varepsilon P)$ be the family of the $Q$-periodic functions in $W_{l o c}^{1, p}(\varepsilon P)$ whose restriction to $Q \cap \varepsilon P$ belongs to $W^{1, p}(Q \cap \varepsilon P)$. Moreover, given $\nu \in \mathbb{S}^{1}$, we denote by $Q^{\nu}$ any unit cube centered at the origin with one face orthogonal to $\nu$ and by $S B V_{0,1}\left(Q^{\nu} \cap \varepsilon P\right)$ the family of functions $w \in S B V\left(Q^{\nu} \cap \varepsilon P\right)$ such that $\nabla w=0 \mathcal{L}^{n}$ a.e. in $Q^{\nu} \cap \varepsilon P$, with $w(x)=1,0$ on a neighborhood of $\partial Q^{\nu} \cap\{x \cdot \nu \geq 0\}$, $\partial Q^{\nu} \cap\{x \cdot \nu<0\}$, respectively.

Let $p \in(1,+\infty)$ and consider a Carathéodory function $f: \mathbb{R}^{n} \times \mathbb{R}^{n} \rightarrow[0,+\infty)$ and a Borel function $g: \mathbb{R}^{n} \times \mathbb{S}^{n-1} \rightarrow[0,+\infty)$. We suppose that $f$ satisfies

(f1) $f(\cdot, \xi)$ is $Q$-periodic for every $\xi \in \mathbb{R}^{n}$,

(f2) there exist two constants $c_{1}, c_{2}>0$ such that for every $(x, \xi) \in \mathbb{R}^{n} \times \mathbb{R}^{n}$

$$
c_{1}|\xi|^{p} \leq f(x, \xi) \leq c_{2}\left(1+|\xi|^{p}\right),
$$

and that $g$ satisfies

(g1) $g(\cdot, \nu)$ is $Q$-periodic for every $\nu \in \mathbb{S}^{n-1}$,

(g2) $g(x,-\nu)=g(x, \nu)$ for every $(x, \nu) \in \mathbb{R}^{n} \times \mathbb{S}^{n-1}$,

(g3) there exist two constants $c_{3}, c_{4}>0$ such that for every $(x, \nu) \in \mathbb{R}^{n} \times \mathbb{S}^{n-1}$

$$
c_{3} \leq g(x, \nu) \leq c_{4}
$$

Then we introduce the family of functionals $\mathcal{F}_{\varepsilon}: L^{p}(\Omega) \rightarrow[0,+\infty]$ defined by

$$
\mathcal{F}_{\varepsilon}(u):= \begin{cases}\int_{\Omega_{\varepsilon}} f\left(\frac{x}{\varepsilon}, \nabla u\right) d x+\int_{S_{u} \cap \Omega_{\varepsilon}} g\left(\frac{x}{\varepsilon}, \nu_{u}\right) d \mathcal{H}^{n-1} & \left.u\right|_{\Omega_{\varepsilon}} \in S B V^{p}\left(\Omega_{\varepsilon}\right), \\ +\infty & \text { otherwise in } L^{p}(\Omega) .\end{cases}
$$

Its asymptotic behavior is described by the following theorem.

Theorem 4. The family $\left(\mathcal{F}_{\varepsilon}\right) \Gamma\left(L^{p}(\Omega)\right)$-converges to the functional $\mathcal{F}_{\text {hom }}: L^{p}(\Omega) \rightarrow[0, \infty]$ given by

$$
\mathcal{F}_{\text {hom }}(u):= \begin{cases}\int_{\Omega} f_{\text {hom }}(\nabla u) d x+\int_{S_{u}} g_{\text {hom }}\left(\nu_{u}\right) d \mathcal{H}^{n-1} & u \in G S B V^{p}(\Omega), \\ +\infty & \text { otherwise in } L^{p}(\Omega),\end{cases}
$$

where the bulk energy density $f_{\text {hom }}: \mathbb{R}^{n} \rightarrow[0,+\infty)$ is given by

$$
f_{\text {hom }}(\xi):=\inf \left\{\int_{Q \cap P} f^{c o}(x, \xi+\nabla w) d x: w \in W_{p e r}^{1, p}(Q \cap P)\right\},
$$

with $f^{c o}$ denoting the convex envelope of $f$, and the surface energy density $g_{\text {hom }}: \mathbb{S}^{n-1} \rightarrow$ $[0,+\infty)$ is given by

$$
g_{\text {hom }}(\nu):=\lim _{\varepsilon \rightarrow 0^{+}} \inf \left\{\int_{S_{w} \cap \varepsilon P} g\left(\frac{x}{\varepsilon}, \nu_{w}\right) d \mathcal{H}^{n-1}: w \in S B V_{0,1}\left(Q^{\nu} \cap \varepsilon P\right)\right\} .
$$

Furthermore, with $c_{2}$ the constant in (f2), $c_{4}$ the constant in (g2) and some constants $c_{1}^{\prime}, c_{3}^{\prime}>0$, it hold

$$
\begin{aligned}
c_{1}^{\prime}|\xi|^{p} & \leq f_{\text {hom }}(\xi) \leq c_{2} \mathcal{L}^{n}(P \cap Q)\left(1+|\xi|^{p}\right) & & \text { for every } \xi \in \mathbb{R}^{n}, \\
c_{3}^{\prime} & \leq g_{\text {hom }}(\nu) \leq c_{4} \mathcal{L}^{n}(P \cap Q) & & \text { for every } \nu \in \mathbb{S}^{n-1} .
\end{aligned}
$$


Proof. The starting point of our analysis are the local methods of $\Gamma$-convergence. For every open subset $A$ of $\Omega$ let $\mathcal{F}_{\varepsilon}(u, A)$ be defined as $\mathcal{F}_{\varepsilon}(u)$ in (4.1), with $\Omega$ replaced by $A$. Then, for any sequence $\left(\varepsilon_{k}\right)$ there exists a subsequence $\left(\varepsilon_{j_{k}}\right)$ for which $\left(\mathcal{F}_{\varepsilon_{j_{k}}}(\cdot, A)\right) \Gamma$-converges to a functional $\mathcal{F}(\cdot, A)$ for every $A$, and such that for every $u \in S B V^{p} \cap L^{p}(A)$

$$
\mathcal{F}(u, A)=\int_{A} f_{0}(\nabla u) d x+\int_{S_{u} \cap A} g_{0}\left(u^{+}-u^{-}, \nu\right) d \mathcal{H}^{n-1},
$$

for some functions $f_{0}: \mathbb{R}^{n} \rightarrow[0,+\infty]$ and $g_{0}: \mathbb{R} \times \mathbb{S}^{n-1} \rightarrow[0,+\infty]$. The former integral representation result is based on an adaptation of $[7$, Theorem 1] performed in [18, Propositions 5.1 and 5.2].

If we show that for any sequence $\left(\varepsilon_{k}\right)$ the functions $f_{0}$ and $g_{0}$ above are given by $f_{\text {hom }}$ and $g_{h o m}$, respectively, then Urysohn property will entail the conclusion. To simplify the notation in the sequel we suppose that the sequence $\left(\mathcal{F}_{\varepsilon_{k}}(\cdot, A)\right)$ is converging itself.

In this respect, let us point out that the limit in (4.3) defining $g_{\text {hom }}$ exists by reproducing the proof of [9, Proposition 2.2] (see [18, Lemma 5.1] in case $g=1$ ). In addition, equality $g_{\text {hom }}=g_{0}$ can be obtained arguing as in [18, Proposition 5.4] (the latter is actually proven under the assumption $g=1$ ).

To conclude we are left with showing equality $f_{\text {hom }}=f_{0}$.

By adapting the first part of the proof of [7, Theorem 4], we have that for every open subset $A$ of $\Omega$ and $u \in W^{1, p}(A)$ the $L^{p}$ lower-semicontinuous envelope of $\mathcal{F}_{\varepsilon}$ is given by

$$
\overline{\mathcal{F}}_{\varepsilon}(u, A)=\int_{A \cap \varepsilon P} f^{c o}\left(\frac{x}{\varepsilon}, \nabla u\right) d x
$$

and by [8, Proposition 7.13]

$$
\Gamma\left(L^{p}(A)\right)-\lim _{k} \overline{\mathcal{F}}_{\varepsilon_{k}}(u, A)=\mathcal{F}(u, A) .
$$

Moreover, by [8, Proposition 19.6 and Remark 19.2] the limit below exists and

$$
f_{\text {hom }}(\xi)=\lim _{\varepsilon \rightarrow 0^{+}} \inf \left\{\int_{Q \cap \varepsilon P} f^{c o}\left(\frac{x}{\varepsilon}, \xi+\nabla w\right) d x: w \in W_{\text {per }}^{1, p}(Q \cap \varepsilon P)\right\} .
$$

In the sequel we will use both the definition and the latter characterization of $f_{\text {hom }}$ to prove the desired equality.

Step 1: $f_{0} \leq f_{\text {hom }}$.

With fixed $\delta>0$, there exists $w_{0} \in W_{\text {per }}^{1, p}(Q \cap P)$ such that

$$
\int_{Q \cap P} f^{c o}\left(x, \xi+\nabla w_{0}(x)\right) d x \leq f_{h o m}(\xi)+\delta .
$$

We regard $w_{0}$ as extended to the whole of $\mathbb{R}^{n}$ by setting it equal to 0 in $\mathbb{R}^{n} \backslash P$. Define $w_{k}(x):=\varepsilon_{k} w_{0}\left(x / \varepsilon_{k}\right)$, then $w_{k} \in L^{p}(Q),\left.w_{k}\right|_{Q \cap \varepsilon_{k} P} \in W^{1, p}\left(Q \cap \varepsilon_{k} P\right)$, and $\left(w_{k}\right)$ converges to 0 in $L^{p}(Q)$ as $k \rightarrow+\infty$. Furthermore, setting $v_{k}(x):=\xi \cdot x+w_{k}(x)$, a simple change of variables and the periodicity assumption (f1) yield

$$
\overline{\mathcal{F}}_{\varepsilon_{k}}\left(v_{k}, Q\right) \leq \varepsilon_{k}^{n}\left(1+\left[\varepsilon_{k}^{-1}\right]\right)^{n} \int_{Q \cap P} f^{c o}\left(x, \xi+\nabla w_{0}(x)\right) d x,
$$

with [.] denoting the integer part of the relevant quantity. By (4.5) we infer

$$
f_{0}(\xi)=\mathcal{F}(\xi \cdot x, Q) \leq \liminf _{k} \overline{\mathcal{F}}_{\varepsilon_{k}}\left(v_{k}, Q\right) \leq f_{\text {hom }}(\xi)+\delta,
$$

and by taking the limit as $\delta \rightarrow 0^{+}$we conclude $f_{0}(\xi) \leq f_{\text {hom }}(\xi)$. 
Step 2: $f_{\text {hom }} \leq f_{0}$.

In this step our arguments differ mostly with respect to those of [18, Step 2 of Proposition 5.3] in which the extension result for Sobolev functions on perforated domains contained in [1, Theorem 2.1] has been employed in case $P$ is Lipschitz regular. Instead, we take advantage of the decomposition result proved in Proposition 3 to deal with such a case. Following [18, Step 3 of Proposition 5.3] an approximation argument is exploited to recover the general case.

Let us first outline the approximation procedure. Let $\left(P^{m}\right)$ be a sequence of open, $Q$-periodic, connected and Lipschitz sets such that $P^{m} \subseteq P^{m+1}$ and $\cup_{m} P^{m}=P$ as in Lemma 8 , and define $f_{\text {hom }}^{m}$ as $f_{\text {hom }}$ in (4.2) with $P$ replaced by $P^{m}$, then we claim that

$$
\sup _{m} f_{\text {hom }}^{m}=f_{\text {hom }} \text {. }
$$

Clearly $f_{\text {hom }}^{m} \leq f_{\text {hom }}^{m+1} \leq f_{\text {hom }}$ by definition; to prove the opposite inequality we denote by $E^{m}, m \in \mathbb{N}$, an open, bounded, and connected set having the cone property such that $Q \cap P^{m} \subseteq E^{m} \subseteq P^{m}$ (see the beginning of the proof of Theorem 1).

For every $m \in \mathbb{N}$ let $w_{m} \in W_{\text {per }}^{1, p}\left(Q \cap P^{m}\right)$ be such that $f_{Q \cap P^{1}} w_{m} d x=0$ and

Since for every fixed $M \in \mathbb{N}$

$$
\int_{Q \cap P^{m}} f^{c o}\left(x, \xi+\nabla w_{m}\right) d x \leq f_{h o m}^{m}(\xi)+\frac{1}{m} .
$$

$$
\sup _{m \geq M} \int_{Q \cap P^{M}} f^{c o}\left(x, \xi+\nabla w_{m}\right) d x \leq \sup _{m} f_{\text {hom }}^{m}(\xi)+1,
$$

the sequence $\left(w_{m}\right)_{m \geq M}$ is bounded in $W^{1, p}\left(E^{M}\right)$ by the Poincaré-Wirtinger inequality in Sobolev spaces ([23, Lemma 1.1.11]). Then, a diagonal argument implies the existence of a subsequence $\left(m_{k}\right)$ such that the sequence $\left(w_{m_{k}}\right)$ is weakly convergent in $W^{1, p}\left(Q \cap P^{M}\right)$ to some function $w$, for every $M \in \mathbb{N}$. Since

$$
\int_{Q \cap P^{M}} f^{c o}(x, \xi+\nabla w) d x \leq \liminf _{k} \int_{Q \cap P^{M}} f^{c o}\left(x, \xi+\nabla w_{m_{k}}\right) d x \leq \sup _{m} f_{\text {hom }}^{m}(\xi)+\frac{1}{M},
$$

by letting $M \rightarrow+\infty$, we infer that $w \in L_{l o c}^{p}(Q \cap P), \nabla w \in L^{p}\left(Q \cap P, \mathbb{R}^{n}\right)$ and

$$
\int_{Q \cap P} f^{c o}(x, \xi+\nabla w) d x \leq \sup _{m} f_{h o m}^{m}(\xi) .
$$

In particular, it is easy to check that the truncated functions $v_{j}:=w \wedge j \vee-j$, extended by $Q$-periodicity, belong to $W_{p e r}^{1, p}(Q \cap P)$ and for every $M \in \mathbb{N}$

$$
\begin{aligned}
& f_{\text {hom }}(\xi) \leq \int_{Q \cap P} f^{c o}\left(\xi+\nabla v_{j}\right) d x \\
& =\int_{(Q \cap P) \backslash\{|w| \geq j\}} f^{c o}(\xi+\nabla w) d x+c_{2}\left(1+|\xi|^{p}\right) \mathcal{L}^{n}((Q \cap P) \backslash\{|w| \geq j\}) \\
& \leq \int_{Q \cap P} f^{c o}(\xi+\nabla w) d x+c_{2}\left(1+|\xi|^{p}\right)\left(\mathcal{L}^{n}\left(\left(Q \cap P^{M}\right) \backslash\{|w| \geq j\}\right)+\mathcal{L}^{n}\left(Q \cap\left(P \backslash P^{M}\right)\right)\right) .
\end{aligned}
$$

Since $w \in L^{p}\left(Q \cap P^{M}\right)$, we have $\mathcal{L}^{n}\left(\left(Q \cap P^{M}\right) \backslash\{|w| \geq j\}\right) \rightarrow 0$ as $j \rightarrow+\infty$, so that

$$
f_{\text {hom }}(\xi) \leq \int_{Q \cap P} f^{c o}(\xi+\nabla w) d x+c_{2}\left(1+|\xi|^{p}\right) \mathcal{L}^{n}\left(Q \cap\left(P \backslash P^{M}\right)\right) .
$$

Gathering (4.8) and (4.9), we obtain (4.7) as $M \rightarrow+\infty$. 
With the help of (4.7) we are able to show that $f_{\text {hom }}(\xi) \leq f_{0}(\xi)$. With fixed $m \in \mathbb{N}$, denote by $\mathcal{F}_{\varepsilon}^{m}$ the functionals defined as $\mathcal{F}_{\varepsilon}$ with $P$ replaced by $P^{m}$. Consider a sequence $\left(u_{k}\right) \subset L^{p}(Q)$ converging to $\xi \cdot x$ in $L^{p}(Q)$ and such that

$$
f_{0}(\xi)=\mathcal{F}(\xi \cdot x, Q)=\lim _{k} \mathcal{F}_{\varepsilon_{k}}\left(u_{k}, Q\right) .
$$

By Proposition 3, there exist a subsequence $\left(k_{j}\right)$ and a sequence $\left(w_{j}\right) \subset W^{1, \infty}(Q)$ such that $w_{j} \rightarrow 0$ strongly in $L^{\infty}(Q), w_{j}=0$ on a neighborhood of $\partial Q,\left(\chi_{Q \cap \varepsilon_{k_{j}} P^{m}} \nabla w_{j}\right)$ is $p$ equiintegrable and $\lim _{j} \mathcal{L}^{n}\left(U_{j}\right)=0$, where $U_{j}:=\left\{x \in Q \cap \varepsilon_{k_{j}} P^{m}: u_{k_{j}}(x) \neq \xi \cdot x+w_{j}(x)\right\}$.

Then, by extending $w_{j}$ to $\mathbb{R}^{n}$ by $Q$-periodicity and by the characterization in (4.6) for $f_{\text {hom }}^{m}$, we get

$$
\begin{aligned}
f_{\text {hom }}^{m}(\xi) & \leq \underset{j}{\limsup } \mathcal{F}_{\varepsilon_{k_{j}}}^{m}\left(\xi \cdot x+w_{j}, Q\right) \leq \limsup _{j} \mathcal{F}_{\varepsilon_{k_{j}}}^{m}\left(u_{k_{j}}, Q\right) \\
& +c_{2} \limsup _{j} \int_{U_{j}}\left(1+\left|\xi+\nabla w_{j}\right|^{p}\right) d x \leq f_{0}(\xi),
\end{aligned}
$$

being $\mathcal{F}_{\varepsilon_{k_{j}}}^{m} \leq \mathcal{F}_{\varepsilon_{k_{j}}}$. Taking the supremum in $m$, by (4.7) we conclude.

Eventually, in order to show (4.4) it suffices to take into account (f2) and (g2), and the lower semicontinuity estimates in (2.3), the growth conditions from above being trivially satisfied.

Finally, as a consequence of Theorem 1 , we have $\Gamma$-lim $\mathcal{F}_{\varepsilon}(u)=+\infty$ whenever $u \notin$ $G S B V^{p} \cap L^{p}(\Omega)$. Moreover, the coerciveness conditions established in (4.4) and a by now standard argument (see [9, Lemma 3.5]) imply the continuity of $\mathcal{F}_{\text {hom }}$ along truncations, so that the integral representation can be extended to $G S B V^{p} \cap L^{p}(\Omega)$.

Remark 3. If $P$ is Lipschitz regular the proof of inequality $f_{\text {hom }} \leq f_{0}$ above greatly simplifies since we can skip the approximation argument. In addition, in such a case it also follows that

$$
f_{\text {hom }}(\xi)=\inf \left\{\int_{Q \cap P} f^{c o}(x, \xi+\nabla w) d x: w \in W_{\text {per }}^{1, \infty}(Q)\right\} .
$$

Remark 4. If $f$ is convex in the second variable, then we do not need to relax $\mathcal{F}_{\varepsilon}$ and we can just assume $f$ Borelian.

Arguing as in Theorem 4 we deduce the classical homogenization theorem for degenerate energies on Sobolev spaces. This method is alternative to that employed by [1] relying on an extension result. In what follows we denote by $\mathcal{E}_{\varepsilon}: L^{p}(\Omega) \rightarrow[0,+\infty]$ the family of functionals defined by

$$
\mathcal{E}_{\varepsilon}(u):= \begin{cases}\int_{\Omega_{\varepsilon}} f\left(\frac{x}{\varepsilon}, \nabla u\right) d x & \left.u\right|_{\Omega_{\varepsilon}} \in W^{1, p}\left(\Omega_{\varepsilon}\right), \\ +\infty & \text { otherwise in } L^{p}(\Omega) .\end{cases}
$$

Theorem 5. The family $\left(\mathcal{E}_{\varepsilon}\right) \Gamma\left(L^{p}(\Omega)\right)$-converges to the functional $\mathcal{E}_{\text {hom }}: L^{p}(\Omega) \rightarrow[0, \infty]$ given by

$$
\mathcal{E}_{\text {hom }}(u):= \begin{cases}\int_{\Omega} f_{\text {hom }}(\nabla u) d x & u \in W^{1, p}(\Omega), \\ +\infty & \text { otherwise in } L^{p}(\Omega),\end{cases}
$$

with $f_{\text {hom }}: \mathbb{R}^{n} \rightarrow[0,+\infty)$ defined in (4.2). 
Proof. For every open subset $A$ of $\Omega$, define $\mathcal{E}_{\varepsilon}(u, A)$ as $\mathcal{E}_{\varepsilon}(u)$ in (4.10), with $\Omega$ replaced by $A$. Then the local methods of $\Gamma$-convergence and the integral representation result in [7, Theorem 2] give that for any sequence $\left(\varepsilon_{k}\right)$ there exists a subsequence $\left(\varepsilon_{j_{k}}\right)$ for which $\left(\mathcal{E}_{\varepsilon_{j_{k}}}(\cdot, A)\right) \Gamma$-converges to a functional $\mathcal{E}(\cdot, A)$ for every $A$, and such that for every $u \in W^{1, p}(A)$

$$
\mathcal{E}(u, A)=\int_{A} f_{0}(\nabla u) d x
$$

for some Borel function $f_{0}: \mathbb{R}^{n} \rightarrow[0,+\infty]$. To deduce that $f_{0}=f_{\text {hom }}$ we argue as in Theorem 4. Finally, $\Gamma-\lim \mathcal{E}_{\varepsilon}(u)=+\infty$ whenever $u \notin W^{1, p}(\Omega)$ by Theorem 2 .

Remark 5. The $\Gamma$-convergence statements in Theorems 4 and 5 are compatible with the addition of Dirichlet boundary conditions on the fix boundary $\partial \Omega \cap \partial \Omega_{\varepsilon}$, provided $\Omega$ is assumed to be Lipschitz regular and the boundary datum $g$ is the trace of a function in $W^{1, p} \cap L^{\infty}(\Omega)$ in the first case, and $W^{1, p}(\Omega)$ in the second (compare with [18, Theorems 6.1 and 7.1] and [2, Lemmata A.3, A.4]).

In the first case, by truncating, we can always assume that the sequence $\left(u_{\varepsilon}\right)$ of almost minimizers is bounded in $L^{\infty}\left(\Omega_{\varepsilon}\right)$ and then, by Theorem 4 , that $\left(\tilde{u}_{\varepsilon}\right)$ converges strongly in $L^{p}(\Omega)$ to some $u \in S B V^{p} \cap L^{\infty}(\Omega)$, which turns out to be the related solution for the minimum problem of the $\Gamma$-limit. In the second case instead we need to suppose that $P$ enjoys the cone property to apply Corollary 1.

Eventually, in the Sobolev case one can consider the addition of Neumann boundary conditions (see [1, Theorem 3.1] and [8, Proposition 19.10]). Also in this case, despite the $\Gamma$-limit is provided only under the assumption that $P$ is connected, to guarantee that the limit of the minimizers is a solution for the minimum problem of the $\Gamma$-limit, we need to assume that $P$ has the cone property in order to apply the last part of Theorem 2 .

\section{Appendix A}

We quote some results which were instrumental in the arguments above and maybe less known. First, we state two Poincaré-Wirtinger type inequalities in $B V$ which are usually stated in the Sobolev space setting. Their extensions follows easily since $W^{1,1}$ is dense in $B V$ with respect to the strict convergence, i.e., the convergence of the functions in $L^{1}$ and of the total variation measures.

Proposition 4. There exists a constant $c=c(n)$ such that for every open, bounded and convex set $E \subseteq \mathbb{R}^{n}$

$$
\int_{E}\left|u(x)-u_{E}\right| d x \leq c \operatorname{diam}(E)|D u|(E),
$$

for every $u \in B V(E)$.

Proposition 5. Let $E \subseteq \mathbb{R}^{n}$ be a bounded, connected and open set having the cone property. Given an open ball $B \subseteq E$, there is a constant $c=c(B, E)$ such that

$$
\int_{E}\left|u(x)-u_{B}\right| d x \leq c|D u|(E)
$$

for every $u \in B V(E)$.

For the proofs in the Sobolev space setting see [22, Theorem 12.30] for the first, and [23, Lemma 1.1.11] for the latter.

Take also note that the constant $c$ in Proposition 5 scales linearly: $c(\varepsilon B, \varepsilon E)=\varepsilon c(B, E)$ for every $\varepsilon>0$. 
Then we quote a truncation result. It is a slightly strengthened statement of Chacon's biting lemma. Several versions are known in literature (see [19, Lemma 1.2]) we recall here the one proved in [16, Lemma A.2].

Lemma 7. Let $\left(l_{k}\right) \subset L^{1}(\Omega)$ be bounded, then for every positive increasing sequence $t_{j} \uparrow+\infty$ there exists a subsequence $\left(l_{k_{j}}\right)$ such that $\left(l_{k_{j}} \wedge t_{j} \vee-t_{j}\right)$ is equi-integrable.

Finally, we state an approximation lemma exploited in Theorem 4.

Lemma 8. Let $P \subseteq \mathbb{R}^{n}$ be a connected, $Q$-periodic open set. Then there exists a sequence of smooth sets $\left(P^{m}\right) \subseteq \mathbb{R}^{n}$ enjoying the same properties, and such that $P^{m} \subseteq P^{m+1}$, $\cup_{m} P^{m}=P$.

Proof. If $P=\mathbb{R}^{n}$ there is nothing to prove. Thus, we may assume that $P^{c} \neq \varnothing$ and $0 \in P$ without loss of generality. Then, being $P Q$-periodic, all the vertices $p_{i}, 1 \leq i \leq 2^{n}$, of the unit cube $Q$ belong to $P$; in addition the distance function dist $\left(\cdot, P^{c}\right)$ is $Q$-periodic, too.

By taking into account that $P$ is open and arcwise connected for any two vertices $p_{i}$, $p_{j}$ with $\left|p_{i}-p_{j}\right|=1$ there exists a continuous curve $\gamma_{i, j}:[0,1] \rightarrow P$ with $\gamma_{i, j}(0)=p_{i}$ and $\gamma_{i, j}(1)=p_{j}$. Let

$$
\tilde{S}:=\cup_{i<j} \gamma_{i, j}([0,1]), \quad S:=\cup_{z \in \mathbb{Z}^{n}}(z+\tilde{S}),
$$

then $\operatorname{dist}\left(S, P^{c}\right)>0$ being $S$ and $P$ both $Q$-periodic.

Consider the connected component $A^{m}$ of the set $D^{m}:=\left\{x \in \mathbb{R}^{n}: \operatorname{dist}\left(x, P^{c}\right)>2^{-m}\right\}$, $m \in \mathbb{N}$ sufficiently big, containing $S$. By construction, $A^{m} \subseteq A^{m+1}$ and $\cup_{m} A^{m} \subseteq P$. On the other hand for any point $x \in P$ there exists a continuous curve $\gamma_{x}:[0,1] \rightarrow P$ joining $x$ itself and a vertex $p$ of $Q$. Hence, $\gamma_{x}([0,1]) \subseteq D^{m}$ for $m \in \mathbb{N}$ satisfying $\operatorname{dist}\left(\gamma_{x}([0,1]), P^{c}\right)>$ $2^{-m}$, and since $p \in S \cap \gamma_{x}([0,1])$ by connectedness $\gamma_{x}([0,1]) \subseteq A^{m}$. Hence, $\cup_{m} A^{m}=P$.

To conclude we regularize $\operatorname{dist}\left(\cdot, P^{c}\right)$ in order to get smooth open sets as in the statement. To this aim, consider a kernel $\varphi \in C_{c}^{\infty}\left(B_{1},[0,+\infty)\right)$ with $\|\varphi\|_{L^{1}\left(\mathbb{R}^{n}\right)}=1$ and define the mollified functions $d_{k}(x):=\left(\operatorname{dist}\left(\cdot, P^{c}\right) * k^{n} \varphi(k \cdot)\right)(x)$. Take note that $d_{k}$ is $Q$-periodic for all $k \in \mathbb{N}$.

Sard's theorem implies that for every $k \in \mathbb{N}$ the sets $D_{k, \eta}:=\left\{x \in \mathbb{R}^{n}: d_{k}(x)>2^{-m}-\eta\right\}$ are $(n-1)$-dimensional manifolds of class $C^{\infty}$ if $\eta \in I_{m}$, with $I_{m}$ a set of full $\mathcal{L}^{1}$ measure in $\left(2^{-(m+2)}, 2^{-(m+1)}\right)$. By uniform convergence of $\left(d_{k}\right)$ to $\operatorname{dist}\left(\cdot, P^{c}\right)$ on $\mathbb{R}^{n}$ we can find a diverging sequence $\left(k_{m}\right)$ such that

$$
\left\|d_{k_{m}}-\operatorname{dist}\left(\cdot, P^{c}\right)\right\|_{L^{\infty}\left(\mathbb{R}^{n}\right)}+\left\|d_{k_{m}}-d_{k_{m+1}}\right\|_{L^{\infty}\left(\mathbb{R}^{n}\right)} \leq 2^{-(m+2)} .
$$

To conclude let $\left(\eta_{m}\right)$ be a decreasing sequence, with $\eta_{m} \in I_{m}$ and $\eta_{m}-\eta_{m+1}<2^{-(m+2)}$, then $D^{m} \subseteq D_{k_{m}, \eta_{m}} \subseteq D_{k_{m+1}, \eta_{m+1}}$. Denote by $P^{m}$ the connected component of $D_{k_{m}, \eta_{m}}$ containing $A^{m}$, the family $\left(P^{m}\right)$ satisfies by construction all the requirements of the statement.

\section{ACKNOWLEDGMENTS}

The research of M. Barchiesi was partially supported by the Center for Nonlinear Analysis (NSF Grants No. DMS-0405343 and DMS-0635983).

M. Focardi acknowledges the kind hospitality of the Center for Nonlinear Analysis where this joint project started during a visit in spring 2008 .

The authors are indebted to Giovanni Leoni for bringing to their attention Proposition 4. 


\section{REFERENCES}

[1] E. Acerbi, V. Chiadò Piat, G. Dal Maso \& D. Percivale. An extension theorem from connected sets, and homogenization in general periodic domains. Nonlinear Anal. 18 (1992), 481-496.

[2] G. Allaire \& F. Murat. Homogenization of the Neumann problem with nonisolated holes. Asymptotic Analysis 7 (1993), 81-95.

[3] L. Ambrosio. On the lower semicontinuity of quasi-convex integrals in $S B V\left(\Omega ; \mathbb{R}^{k}\right)$. Nonlinear Anal. 23 (1994), 405-425.

[4] L. Ambrosio \& A. Braides. Energies in SBV and variational models in fracture mechanics, in "Homogenization and applications to material sciences", Nice (1995), 1-22. GAKUTO Internat. Ser. Math. Sci. Appl., vol. 9, Gakkōtosho, Tokyo, 1995.

[5] L. Ambrosio, N. Fusco \& D. Pallara. Functions of bounded variation and free discontinuity problems, in the Oxford Mathematical Monographs. The Clarendon Press Oxford University Press, New York, 2000 .

[6] M. Barchiesi \& G. Dal Maso. Homogenization of fiber reinforced brittle materials: the extremal cases. SIAM J. Math. Anal. 41 (2009), 1874-1889.

[7] G. Bouchitté, I. Fonseca, G. Leoni \& L. Mascarenhas. A global method for relaxation in $W^{1, p}$ and in SBV ${ }^{p}$. Arch. Rat. Mech. Anal. 165 (2002), 187-242.

[8] A. Braides \& A. Defranceschi. Homogenization of Multiple integrals. Oxford University Press, Oxford, 1998.

[9] A. Braides, A. Defranceschi \& E. Vitali. Homogenization of free discontinuity problems. Arch. Rat. Mech. Anal. 135 (1996), 297-356.

[10] A. Braides \& A. Garroni. Homogenization of periodic nonlinear media with stiff and soft inclusions. Math. Models Methods Appl. Sci. 5 (1995), 543-564.

[11] H. Brezis. Analyse Fonctionnelle - Théorie et applications. Masson, Paris, 1983.

[12] M. Briane. A spectral approach for the homogenization in general periodically perforated domains in Homogenization, Naples (2001), 219-224, GAKUTO Internat. Ser. Math. Sci. Appl., vol. 18. Gakkōtosho, Tokyo, 2003.

[13] M. Briane, A. Damlamian \& P. Donato. H-convergence for perforated domains in Nonlinear partial differential equations and their applications Collège de France Seminar, Vol. XIII (Paris, 1994/1996), 62-100. Pitman Res. Notes Math. Ser., vol. 391. Longman, Harlow, 1998.

[14] F. Cagnetti \& L. Scardia. An extension theorem in $S B V$ and an application to the homogenization of the Mumford-Shah functional in perforated domains. J. Math. Pures Appl. (9), to appear.

[15] E. De Giorgi, M. Carriero \& A. Leaci. Existence theorem for a minimum problem with free discontinuity set. Arch. Ration. Mech. Anal., 108 (1989), 195-218.

[16] C. De Lellis, M. Focardi \& E. Spadaro. Lower semicontinuous functionals for Almgren's multiple valued functions. Preprint 2009.

[17] L.C. Evans \& R.F. Gariepy. Measure theory and fine properties of functions, Studies in Advanced Mathematics. CRC Press, Boca Raton, FL, 1992.

[18] M. Focardi, M.S. Gelli \& M. Ponsiglione. Fracture mechanics in perforated domains: a variational model for brittle porous media. Math. Models Methods Appl. Sci. 19 (2009), 2065-2100.

[19] I. Fonseca, S. Müller \& P. Pedregal. Analysis of concentration and oscillation effects generated by gradients. SIAM J. Math. Anal. 29 (1998), 736-756.

[20] J. Kristensen. Lower semicontinuity in spaces of weakly differentiable functions. Math. Ann., 313 (1999), 653-710.

[21] C.J. Larsen. On the representation of effective energy densities. ESAIM Control Optim. Calc. Var. 5 (2000), 529-538.

[22] G. Leoni. A first course in Sobolev spaces, in the Graduate Studies in Mathematics, vol. 105. American Mathematical Society, Providence, 2009.

[23] V.G. Maz'ja. Sobolev spaces, in the Springer Series in Soviet Mathematics. Springer-Verlag, Berlin, 1985.

[24] E. M. Stein. Singular integrals and differentiability properties of functions. in the Princeton Mathematical Series, vol. 30. Princeton University Press, N.J., 1970.

[25] Tartar, L. The general theory of homogenization: a personalized introduction, in the Lecture Notes of the Unione Matematica Italiana, vol. 7. Springer-Verlag, Berlin, 2009. 
(M. Barchiesi) BCAM, Bizkaia Technology Park, Building 500, 48160 Derio, Spain E-mail address: barchiesi@bcamath.org

(M. Focardi) Università di Firenze, Viale Morgagni 67/A, I-50134 Firenze, Italy E-mail address: focardi@math.unifi.it 